\title{
Neural networks based predictive control for thermal comfort and energy savings in public buildings
}

\author{
P.M. Ferreira ${ }^{b, c, *}$, A.E. Ruano ${ }^{a, c}$, S. Silva ${ }^{a}$, E.Z.E. Conceição ${ }^{a}$ \\ a University of Algarve, 8005-139 Faro, Portugal \\ ${ }^{\mathrm{b}}$ Algarve Science and Technology Park, Campus de Gambelas, Pav. A5, 8005-139 Faro, Portugal \\ ${ }^{c}$ Centre for Intelligent Systems, IDMEC-IST, Av. Rovisco Pais 1, 1049-001 Lisboa, Portugal
}

\section{A R T I C L E I N F O}

\section{Article history:}

Received 17 February 2012

Received in revised form 17 May 2012

Accepted 2 August 2012

\section{Keywords:}

HVAC predictive control

Predicted mean vote

Neural networks

Multi-objective genetic algorithm

Thermal comfort

Wireless sensor networks

\begin{abstract}
A B S T R A C T
The paper addresses the problem of controlling a Heating Ventilation and Air Conditioning (HVAC) system with the purpose of achieving a desired thermal comfort level and energy savings. The formulation uses the thermal comfort, assessed using the predicted mean vote (PMV) index, as a restriction and minimises the energy spent to comply with it. This results in the maintenance of thermal comfort and on the minimisation of energy, which in most conditions are conflicting goals requiring an optimisation method to find appropriate solutions over time. A discrete model-based predictive control methodology is applied, consisting of three major components: the predictive models, implemented by radial basis function neural networks identified by means of a multi-objective genetic algorithm; the cost function that will be optimised to minimise energy consumption and maintain thermal comfort; and the optimisation method, a discrete branch and bound approach. Each component will be described, with special emphasis on a fast and accurate computation of the PMV indices. Experimental results obtained within different rooms in a building of the University of Algarve will be presented, both in summer and winter conditions, demonstrating the feasibility and performance of the approach. Energy savings resulting from the application of the method are estimated to be greater than $50 \%$.
\end{abstract}

(c) 2012 Elsevier B.V. All rights reserved.

\section{Introduction}

In European Union (EU) countries, primary energy consumption in buildings represents about $40 \%$ of the total energy consumption and, with variations from country to country, half of this energy is spent for indoor climate conditioning [1-3]. It is estimated that the use of efficient energy management systems in buildings can save up to $8 \%$ of the energy consumption in the entire EU [4]. Around $83 \%$ of the EU dwellings were constructed before 1990 and about $50 \%$ of them before 1970 [1]. Therefore it is of fundamental importance to control efficiently the existing HVAC systems, in order to decrease energy usage and increase compliance with the European Directive (2010/31/EU) on the energy performance of buildings [3].

The use of Artificial Neural Networks (ANNs) in various applications related with energy management in buildings has been increasing significantly over the recent years. Within this area, ANNs have been mainly applied in several aspects of HVAC control

\footnotetext{
* Corresponding author at: Algarve Science and Technology Park, Campus de Gambelas, Pav. A5, 8005-139 Faro, Portugal. Tel.: +351 289800950; fax: +351289800098. E-mail addresses: pfrazao@ualg.pt (P.M. Ferreira), aruano@ualg.pt (A.E. Ruano), ssilva@ualg.pt (S. Silva), econcei@ualg.pt (E.Z.E. Conceição).
}

methodologies [5-11], and in forecasting energy consumption [12-20].

The authors have advocated, in past publications [21,22], the use of model based predictive control (MBPC) with the purpose of efficiently controlling existing HVAC systems in large public buildings. The present paper is the natural follow-up of this past work, where a remote, wired data acquisition system was set-up in a secondary school in the South of Portugal. The performance of physical models, based on energy and mass balance integral-differential equations were evaluated against black-box, neural network models for the purpose of modelling the inside air temperature. It was found that the role of these two different types of models is complementary, and not competing. While physical models can be used in the phase of the project of buildings, and to assess the consequences of possible buildings modifications, data-driven models such as neural networks should be used for the on-line control of HVAC systems. Simulations showed the potential of MBPC for the control of air-conditioned systems, indicating both the better regulation and the energy savings obtained.

This article is organised as follows. The experimental setup is described in Section 2. As a PMV formulation is employed, its computation is discussed in Section 3. To implement the MBPC strategy, several neural network models are used. Their design is briefly addressed in Section 4 . Section 5 describes the branch \& bound (BB) 


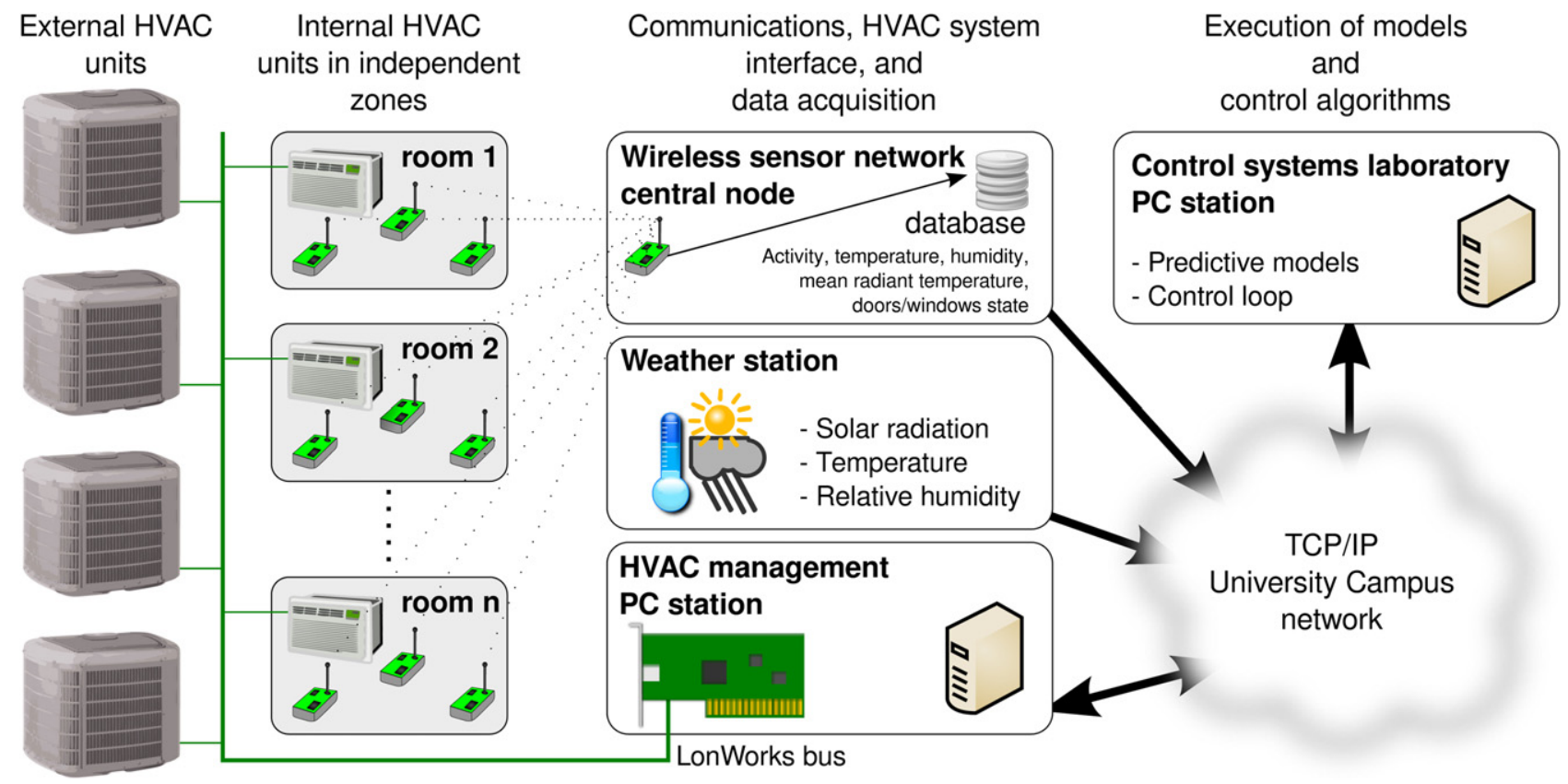

Fig. 1. Overview of the experimental set up used.

search technique that is employed to implement the MBPC, which in turn is formulated in Section 6. Several experiments are then presented in Section 7, both in summer and winter conditions. The paper ends with conclusions and a description of future work.

\section{Experimental setup}

HVAC control experiments were conducted in three areas of one building used by the Faculty of Sciences \& Technology of the University of Algarve, in the south of Portugal. In total, 16 locations (labs, offices, corridors) are now equipped with data acquisition devices and their internal HVAC units may be independently controlled with new algorithms, and monitored. For the work that is going to be presented, four rooms were used, denoted by the letters A, B, C, and D. Rooms A and B are adjacent with walls facing west and north (only A). Room C shares the same corridor with A and B and has walls exposed to the north and east. These three rooms are on the second floor. Finally, room D is directly below room C with walls exposed to the north and east.

A weather station located in the campus provides air temperature $\left(T_{a o}\right)$, air humidity $\left(H_{a o}\right)$, and global solar radiation $\left(R_{s g}\right)$ measurements. All the elements involved are connected to the TCP/IP network, enabling a PC station in the control systems laboratory to monitor and control any of the rooms. Fig. 1 provides an illustration of the system integration.

\subsection{Wireless sensor networks}

WSNs are rapidly gaining popularity, and are being used in a vast spectrum of applications. One of the most active area being building automation $[23,24]$. Each of the rooms where the experiments were conducted has WSN nodes with sensors to monitor the air temperature $\left(T_{a i}\right)$, air humidity $\left(H_{a i}\right)$, globe temperature $\left(T_{g}\right)$, the state of windows and doors (open/closed), and movement using a passive infra-red activity monitor. $T_{g}$ is measured by means of a black globe thermometer [25] and its measurement is then used to determine the mean radiant temperature, $T_{m r}$ [26].

The WSNs have a star topology, where each unit is collecting information once per minute and sending it to a central node with storage and database capabilities. Each node consists of one Tmote Sky platform connected to the required sensors. This platform is an IEEE 802.15.4 standard compliant device that uses the TinyOS [27] operating system.

In each room one WSN node is installed in the wall, nearly $1.75 \mathrm{~m}$ above the floor, which measures $T_{a i}$ and $H_{a i}$ using SHT11 sensors from Sensirion. Approximately in the middle of the room, at a height of roughly $2 \mathrm{~m}$ above the floor (for security reasons), the black globe thermometer is installed. It consists of a mate black painted sphere with a diameter of $125 \mathrm{~mm}$ with a Tmote (and its temperature sensing device) at its centre. Plastic was used instead of copper, which is more commonly found in this application, because it was easily available, but also because according to [26] it overcomes an undesirable high time constant that appears when copper is employed. Infra-red sensors are installed at one corner of the room to monitor activity. Digital information, both from the magnetic switches in doors and windows and from the movement detector are wired to one of the existing WSN nodes.

\subsection{HVAC system}

The HVAC system used in the experiments is composed of 3 independent Mitsubishi Variable Refrigerant Flow (VRF) systems, each one with an outdoor air cooled inverter compressor unit (denoted in the sequel as outdoor unit), located on the building roof, connected to ceiling concealed ducted indoor units (denoted as interior units). In each independent room there is at least one internal unit, with its own wall controller. The system can be centrally managed by a PC management station to which all the units are connected via a LonWorks communication bus. This station is able to monitor and control many aspects of all the HVAC system, through the Mitsubishi LMAP02 interface [28]. Those of major interest for the experiments are: specifying a temperature set-point for a given unit, switching the internal unit on or off, and disabling the local controller so the occupants cannot operate the internal unit while experiments are being conducted.

The model of the interior units used in the experiments described in this paper is the PEFY-P63VMM and the model of the outdoor units is the PUHY-250YMF-C. 


\section{Predicted mean vote}

The holly grail of energy management in buildings is the minimisation of the energy required to maintain a desired minimum comfort level for the occupants. Although the perception of comfort is related to several environmental factors such as lighting, temperature and air quality, in this work only the thermal comfort conditions aspect is addressed. As a discrete MBPC problem, described in Section 5, is solved using the BB search technique, finding the optimal solution depends on the ability of computing many PMV values in a small amount of time. As the PMV formulation involves iterative computations consuming variable time, it is crucial to have a method for fast, possibly constant execution time, computation of the PMV index.

Feed-forward ANNs are direct input-to-output connectionist computing structures capable of approximating a smooth function with arbitrary accuracy provided sufficient neurons are used. These features are the means to achieve the requirements stated above. The feed-forward ANN direct input-to-output structure provides the constant execution time, their ability to approximate non-linear functions provide the capability of approximating the PMV function. The accuracy of the approximation is related to the number of neurons used in the ANN hidden layer(s), which in turn is linearly related to the execution time. Consequently the problem consists in finding the appropriate trade-off between the PMV index approximation accuracy and the estimation execution time.

The application of an ANN to estimate the PMV index function has been studied before [29-31]. In all cases the approach taken was not the best for real-time control applications although this was the main motivation. In this work it is shown that by following a simpler and more appropriate approach, it is possible to select a desired compromise between accuracy and execution time. More importantly it is shown that, when compared to the results in [29-31], an increased accuracy with shorter execution time may be achieved.

\subsection{The PMV index}

The American Society of Heating Refrigerating and Air Conditioning Engineers (ASHRAE) proposed the thermal sensation scale with the purpose of quantifying the thermal sensation of people [32]. It uses an integer numerical coding to express the qualitative thermal sensation, by relating the integer range $[-3,3]$ to the qualitative words cold, cool, slight cool, neutral, slight warm, warm, and hot.

An index, designated PMV, was proposed by Fanger [33] in order to predict the average vote of a large group of persons on the thermal sensation scale. It depends on six factors: metabolic rate, clothing insulation, air temperature and humidity, air velocity, and the mean radiant temperature. It is computed by means of a heat-balance equation $[34,35]$ given by,

$$
\begin{aligned}
& P M V=\left(0.303 \mathrm{e}^{-0.036 M}+0.028\right)\left[(M-W)-3.05 \times 10^{-3}\right. \\
& \quad \times\left[5733-6.99(M-W)-P_{a}\right]-0.42[(M-W)-58.15] \\
& -1.7 \times 10^{-5} M\left(5867-P_{a}\right)-0.0014 M\left(34-T_{a i}\right)-3.96 \times 10^{-8} f_{c l} \\
& \left.\quad \times\left[\left(T_{c l}+273\right)^{4}-\left(\overline{T_{r}}+273\right)^{4}\right]-f_{c l} h_{c}\left(T_{c l}-T_{a i}\right)\right],
\end{aligned}
$$

where $M$ and $W$ are the metabolic rate and external work, both in $\mathrm{W} / \mathrm{m}^{2}, P_{a}$ is the partial water vapour pressure in Pascal, and $T_{a i}$ and $\overline{T_{r}}$ are the air temperature and mean radiant temperature, in ${ }^{\circ} \mathrm{C}$. The surface temperature of clothing, $T_{c l}$, and the convective heat transfer coefficient, $h_{c}$, are given by,

$$
\begin{aligned}
& T_{c l}=35.7-0.028(M-W)-I_{c l}\left[3.96 \times 10^{-8} f_{c l} \times\left[\left(T_{c l}+273\right)^{4}\right.\right. \\
& \left.\left.\quad-\left(\overline{T_{r}}+273\right)^{4}\right]+f_{c l} h_{c}\left(T_{c l}-T_{a i}\right)\right]
\end{aligned}
$$

and

$h_{c}=\left\{\begin{array}{ll}h_{c}^{*} & \text { if } h_{c}^{*}>12.1 \sqrt{V_{a}} \\ 12.1 \sqrt{V_{a}} & \text { if } h_{c}^{*}<12.1 \sqrt{V_{a}}\end{array} \quad\left(h_{c}^{*}=2.38\left(T_{c l}-T_{a i}\right)^{1 / 4}\right)\right.$,

respectively. $V_{a}$ is the air velocity in $\mathrm{m} / \mathrm{s}$ and $I_{c l}$ is the clothing thermal resistance in $\mathrm{m}^{2}{ }^{\circ} \mathrm{C} / \mathrm{W}$. These two equations are solved iteratively until a prescribed degree of convergence is attained or a maximum number of iterations is reached. Finally, in (1) and (2), $f_{c l}$, which is the ratio of body surface area covered by clothes to the naked surface area, is defined by:

$f_{c l}= \begin{cases}1.00+1.290 I_{c l} & \text { if } I_{c l} \leq 0.078 \\ 1.05+0.645 I_{c l} & \text { if } I_{c l}>0.078\end{cases}$

The mean radiant temperature, $\overline{T_{r}}$, is a quantity which is hard to measure. The instrument most commonly employed in its determination is a black globe thermometer [25]. Denoting the globe temperature by $T_{g}$, the mean radiant temperature may be determined as [26],

$\overline{T_{r}}=\left[\left(T_{g}+273\right)^{4}+\frac{1.10 \times 10^{8} V_{a}^{0.6}}{\epsilon D^{0.4}}\left(T_{g}-T_{a i}\right)\right]^{1 / 4}-273$,

where $D$ and $\epsilon$ are the globe diameter in meters and the globe emissivity coefficient, respectively.

$P_{a}$, the water vapour pressure in Pascal, is easily related to the relative humidity of the air, $H_{a i}$, by means of Antoine's equation [36]:

$P_{a}=10 H_{a i} \mathrm{e}^{\left(16.6536-4030.183 /\left(T_{a i}+235\right)\right)}$

Using (1)-(6), the PMV appears conceptually as a function of six variables that can be measured or estimated:

$P M V=f\left(T_{a i}, T_{g}, H_{a i}, V_{a}, I_{c l}, M\right)$

\subsection{Proposed approach for real-time control}

Previous ANN models of the PMV index found in the literature employ multi-layer perceptron networks $[29,31]$ or Least-Squares Support Vector Machines (LSSVM) [30].

For real-time control applications there are two important features that any PMV index approximation method should efficiently balance: accuracy and computing time. This means that the PMV models should simultaneously be as simple and as accurate as possible. Although the motivation of past works [29-31] was their use in real-time control systems and the decrease in PMV index computing time, their input/output structure did not optimise properly that balance, as it will be shown later.

For most HVAC real-time control applications, the environment is controlled in closed spaces where all occupants are assumed to be dressed similarly regarding the type of clothing they wear. Moreover it is likely that within each type of closed space they will be performing similar activities like attending a lecture, sitting writing a research paper, or having breakfast at the cafeteria. These two assumptions mean that for a given space it is possible to specify the values of the clothing insulation, $I_{c l}$, and the metabolic rate, $M$, therefore these may be removed from the PMV model input. If it is further assumed that the air velocity, $V_{a}$, varies little within the space and its value is determined by measurements, $V_{a}$ may be considered constant and may also be removed from the PMV model input.

By defining a context vector $C=\left\{I_{c l}, M, V_{a}\right\}$ and by using (1)-(6), a set of input-output data pairs may be generated in order to train an ANN model for the PMV index in the context $C$. This approach suggests that within an HVAC control system like the one in Fig. 1, 


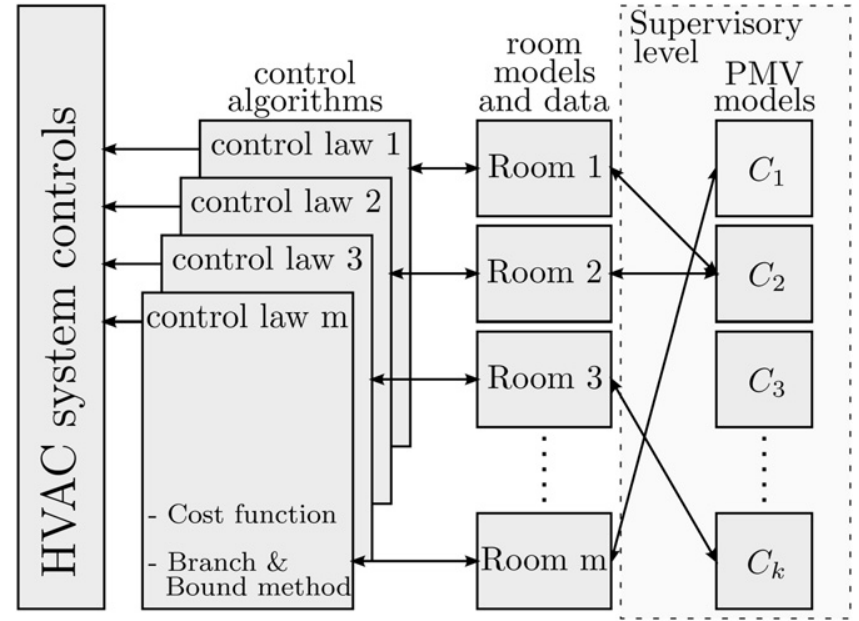

Fig. 2. Using a set of PMV models in an HVAC control system.

there are a set of distinct PMV models, $P M V_{i}$, each for a distinct context $C_{i}$. In this scheme, a supervisory system at an upper operational level will define the correspondence between each room controlled by HVAC systems and a pair $\left\{P M V_{i}, C_{i}\right\}$. The approach is illustrated in Fig. 2. The matching between the rooms and the assumed contexts can be done on the basis of the year season, of the purpose of the room, and maybe of any strong deviations of the outside weather from what is expected.

The consequence of using multiple PMV index models, is that for a specific context $C_{i}$, the model has increased accuracy and reduced computing time when compared to models considering multiple contexts. The increase in accuracy comes from the fact that there are less features to learn in the training data, whereas the decrease in computing time results from using fewer inputs and from the necessity to employ fewer neurons in the hidden layers in order to achieve a desired accuracy. In our case, the air velocity in all rooms considered was measured by a BABUC probe in a $6 \times 6$ grid with a spacing of one meter, at a height of 1.2 meters above ground, and during a period of $3 \mathrm{~min}$. All the measurements were in the range $[0.04,0.16] \mathrm{m} / \mathrm{s}$. The small values of air velocity are justified because the air flow from each indoor unit passes through an air diffuser (DFU 36, from France Air). From these results an average value of approximately $0.08 \mathrm{~m} / \mathrm{s}$ was selected as a constant value to be used in the context vector of PMV index models. Regarding the two additional human factors in the context vector, a value of $69.78 \mathrm{~W} / \mathrm{m}^{2}(1.2 \mathrm{Met})^{1}$ was selected as the metabolic rate of a sedentary activity (see appendix A of [32]), and for the clothing insulation a value of 0.85 clo was used (see appendix B of [32]) in the following experiences.

\subsection{Radial basis function neural network}

The RBF ANN was used in this work as a function approximator to the PMV index (1). The RBF models are trained using the Levenberg-Marquardt (LM) algorithm $[37,38]$ minimising a modified training criterion $[39,40]$.

RBF ANNs have the form,

$\hat{y}(\mathbf{x}, \mathbf{w}, \mathbf{C}, \boldsymbol{\sigma})=\sum_{i=0}^{n} w_{i} \varphi_{i}\left(\mathbf{x}, \mathbf{c}_{i}, \sigma_{i}\right)$,

\footnotetext{
${ }^{1}$ Metabolic equivalent of task (Met). $1 \mathrm{Met}=58.15 \mathrm{~W} / \mathrm{m}^{2}$.
}

where typically $\varphi_{i}$ is the Gaussian function,

$\varphi_{i}\left(\mathbf{x}, \mathbf{c}_{i}, \sigma_{i}\right)=e^{-\left(1 / 2 \sigma_{i}^{2}\right)\left\|\mathbf{x}-\mathbf{c}_{i}\right\|^{2}}, \quad \varphi_{0}=1$.

For a specified number of neurons, $n$, and for a determined set of inputs, $\mathbf{X}^{t}$, off-line training a RBF NN corresponds to determining the values of $\mathbf{w}, \mathbf{C}$, and $\sigma$ such that (10) is minimised:

$\Phi\left(\mathbf{X}^{t}, \mathbf{w}, \mathbf{C}, \boldsymbol{\sigma}\right)=\frac{1}{2}\left\|\mathbf{y}-\hat{\mathbf{y}}\left(\mathbf{X}^{t}, \mathbf{w}, \mathbf{C}, \boldsymbol{\sigma}\right)\right\|^{2}$.

Please note that (10) is now applied to a set of training input patterns, $\mathbf{X}^{t}$, and not to a single input pattern, $\mathbf{x}$. As the model output is a linear combination of the neuron activation functions output (8), (10) can be given as,

$\Phi\left(\mathbf{X}^{t}, \mathbf{w}, \mathbf{C}, \boldsymbol{\sigma}\right)=\frac{1}{2}\left\|\mathbf{y}-\boldsymbol{\phi}\left(\mathbf{X}^{t}, \mathbf{C}, \boldsymbol{\sigma}\right) \mathbf{w}\right\|^{2}$,

where omitting the dependence of $\boldsymbol{\varphi}$ on $\mathbf{C}$ and $\sigma$,

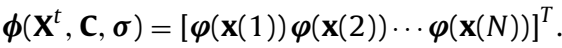

By computing the global optimum value $\left(\mathbf{w}^{*}\right)$ of the linear parameters $\mathbf{w}$, with respect to the nonlinear parameters $\mathbf{C}$ and $\boldsymbol{\sigma}$, as a least-squares solution,

$\mathbf{w}^{*}=\boldsymbol{\phi}^{+}\left(\mathbf{X}^{t}, \mathbf{C}, \boldsymbol{\sigma}\right) \mathbf{y}$

where "+" denotes a pseudo-inverse operation, and replacing (12) in (11), the training criterion to determine the nonlinear parameters $\mathbf{C}$ and $\boldsymbol{\sigma}$ is:

$\Psi\left(\mathbf{X}^{t}, \mathbf{C}, \boldsymbol{\sigma}\right)=\frac{1}{2}\left\|\mathbf{y}-\boldsymbol{\phi}\left(\mathbf{X}^{t}, \mathbf{C}, \boldsymbol{\sigma}\right) \boldsymbol{\phi}^{+}\left(\mathbf{X}^{t}, \mathbf{C}, \boldsymbol{\sigma}\right) \mathbf{y}\right\|^{2}$.

The initial values for the neuron centre positions are randomly selected from the training data, and the spreads of the neuron activation functions are initialised using the simple rule in [41, p. 299]. The training procedure progresses iteratively using the LM algorithm minimising criterion (13), until a termination criterion is satisfied. For more details about the training algorithm and the training criterion the reading of $[40,42-44]$ is recommended.

\subsection{Data sets and model structure}

The input matrix for training the RBF ANNs, defined as $\mathbf{X}^{t}=$

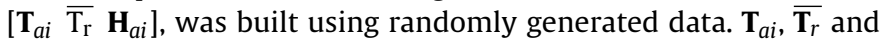
$\mathbf{H}_{a i}$ are vectors of $N$ values taken from the ranges [16, 32], [13, 35] and $[20,70]$, respectively. They were constructed as follows:

1. $\mathbf{T}_{a i}$ and $\mathbf{H}_{a i}$ were selected randomly from a uniform distribution of values in the ranges specified;

2. For each value $T_{a i k}$ in $\mathbf{T}_{a i}$, a corresponding value $T_{g_{k}}$ in $\mathbf{T}_{g}$ was generated using,

$T_{g_{k}}=T_{a i k}+\rho(-3.0,3.0)$,

where $\rho(a, b)$ is a random number from the uniform distribution in the range $[a, b]$. Implicitly it is assumed that $T_{a i}-3<T_{g}<T_{a i}+3$;

3. $\overline{\mathbf{T}_{r}}$ was obtained by means of (5), considering $\mathbf{T}_{a i}$ and $\mathbf{T}_{g}$ just described, $V_{a}=0.08, D=0.125$, and $\epsilon=0.95$.

In order to determine the corresponding output PMV index values, the context vector was defined as described before, i.e., as $C=\{0.85,1.2,0.08\}$. Then, $\mathbf{Y}^{t}$ was constructed by means of (1), using each triplet $X_{k}^{t}$ in $\mathbf{X}^{t}$ along with the values in $C$. This procedure gives rise to the training data set, $\mathbf{D}^{t}=\left\{\mathbf{X}^{t}, \mathbf{Y}^{t}\right\}$.

Using the approach just described, an additional data set, $\mathbf{D}^{v}$, was prepared in order to validate the models with unseen data, after the training stage. $\mathbf{D}^{v}$ has 23,100 training pairs $(N=23,100)$. 

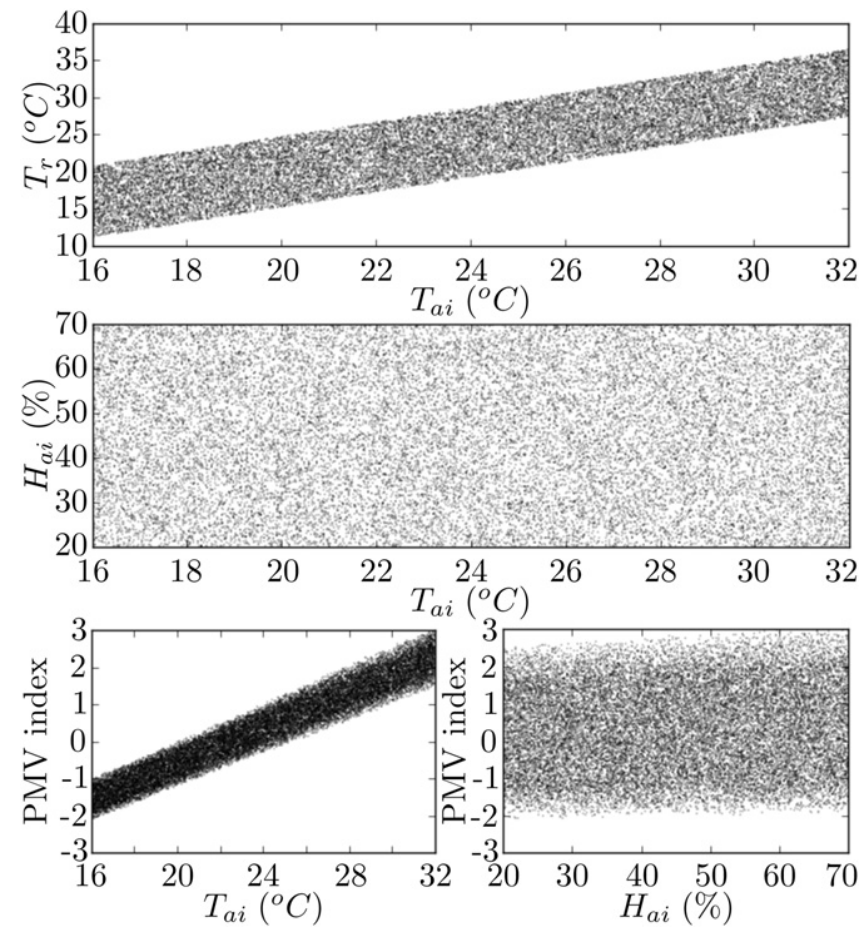

Fig. 3. Input-output variables distribution within the ranges of values considered. The plots correspond to data set $\mathbf{D}^{v}$.

Fig. 3 shows how the input-output patterns are distributed within the ranges specified for the input variables, considering the data in $\mathbf{D}^{v}$ for the context $C$. It may be seen on the top plot how $\overline{T_{r}}$ distributes densely around the values of $T_{a i}$ for the whole range considered.

The same is visible in the middle plot for the relative humidity and air temperature. The bottom plots, from left to right, illustrate the scattering of PMV values around the air temperature and relative humidities, respectively. This data set allows good model evaluation as it efficiently covers the variability of combinations that occurs between input variables.

Although the input-output structure of the model has been specified, there are still two design parameters that need to be determined: the number of neurons and the number of training patterns. For the first, an exhaustive search was conducted over the range [2, 32]. For the second, a search was also conducted as described in the following. It is known that the "ideal" number of training patterns is, to some extent, related to the number of parameters of the model being fitted $[45,46]$. Considering the RBF in (8) for the PMV index model presented, each neuron accounts for 5 parameters, therefore the total number of parameters is given by $n \times 5, n$ being the number of neurons employed. By specifying a number of patterns $(p)$ per model parameter it becomes possible to determine the value of $N$ for the training data set $\mathbf{D}^{t}$, as $N=n \times 5 \times p$. In this case a search was made for $p$ in $\{20,40,60,80,100,120\}$. For each of these values, $n$ was varied in the range $[2,32]$ as already mentioned. Considering that the RBF parameters are initialised randomly, 20 trials were executed for each $(n, p)$ pair. Each trial consisted on the application of the modified training criterion LM algorithm for 200 iterations.

\subsection{Results for the PMV model}

Having determined the models spanned by $\left.(n, p)_{r}\right|_{r=1} ^{20}$, where $r$ is the trial number, the first result sought was a decision on the number of training patterns. The decision was made on the basis of the
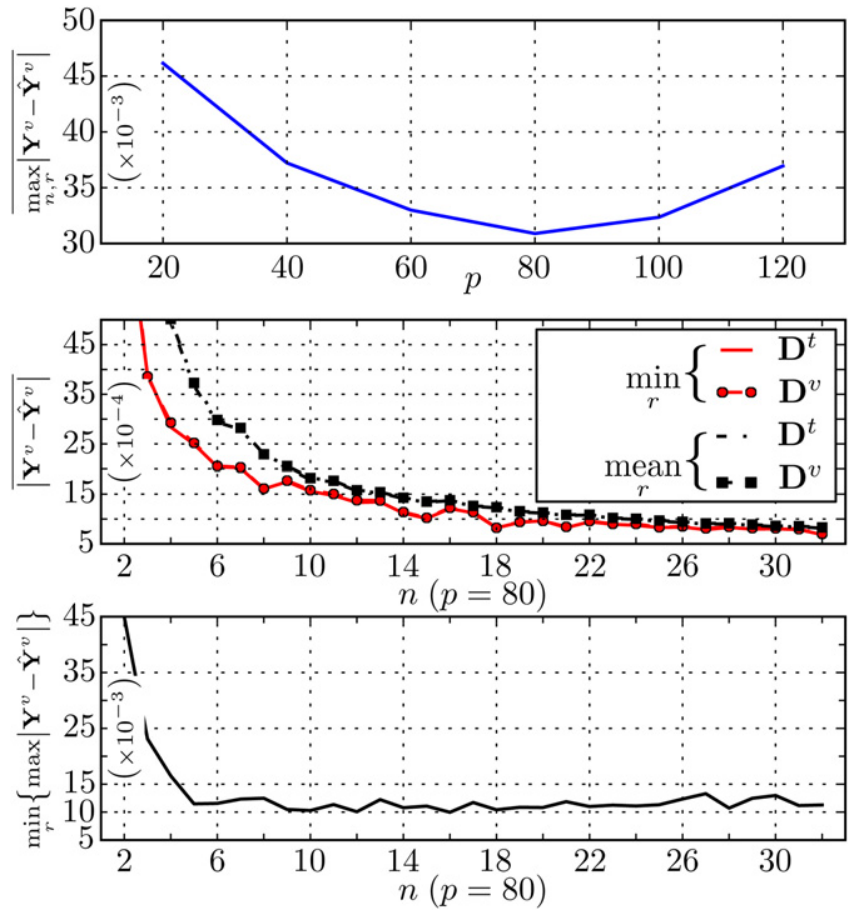

Fig. 4. Results regarding the selection of the best training set size, the generalisation capacity of the models, and the accuracy achieved.

models maximum absolute error obtained on the validation data set, $\mathbf{D}^{v}$. For each value of $p$, the average of that error was computed over all the models (for all $n$ and $r$ ). The top plot of Fig. 4 illustrates the result, where it is clearly seen that, on average, it is best to train the models with 80 training patterns for each model parameter.

The middle plot illustrates the best relation between the results obtained with the training set and those obtained with the validation set, for $p=80$. The plot presents the minimum and mean of the average absolute error obtained over the 20 trials for each number of neurons $n$. The difference is negligible, which allows to conclude that the models provide excellent generalisation capability. To this respect, it should be noted that the validation data set has 23,100 points, a value comparable to the training set in [29], and that with only 1600 training patterns (4 neurons case), near $25 \%$ of the number used in [30], a maximum absolute error of approximately 0.015 is obtained both in training and validation data sets. Still for $p=80$, as it showed the best generalisation, the lower plot in Fig. 4 shows for each number of neurons, the minimum value, obtained over the 20 trials, of the maximum absolute error in the validation data set. It may be concluded that using more than 10 neurons is not necessary as no significant improvements are obtained.

The model with 5 neurons in the lower plot of Fig. 4, corresponding to 26 parameters, achieves an average and maximum absolute error of 0.0025 and 0.011 , respectively. These values are comparable to the results in [30], 0.0022 and 0.0097 (obtained with 100 testing points), although the RBF model is incomparably smaller. Only two results are presented in [29]: a Sum of the Square of the Errors (SSE) on the training set of 0.11 , and a figure showing how the model with 97 parameters behaved on a $9 \mathrm{~h}$ experiment within a room. Regarding the first it is a bit less than half the value of 0.23 obtained by the 5 neuron model. Regarding the figure we may only comment that the well visible errors clearly show that the model does not generalise properly, probably due to a large training set and also, possibly, due to overtraining, considering the small value of SSE. 


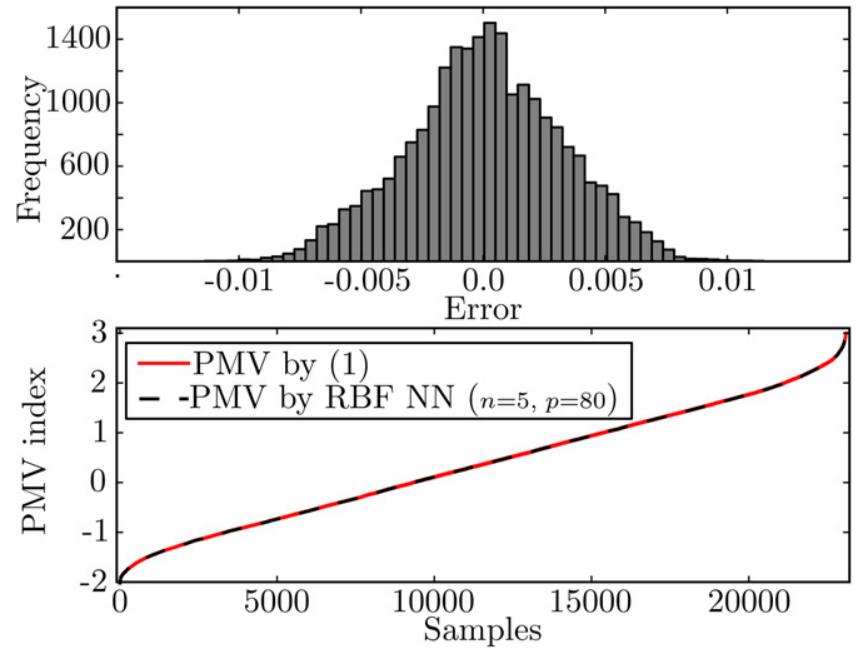

Fig. 5. PMV index fitting on validation data set, obtained with a 5 neuron RBF model. Histogram of error at the top plot.

Fig. 5 shows the fitting of the validation data set by the 5 neurons RBF model, as well as the histogram of the error obtained.

In order to provide a more realistic evaluation of the PMV index model, it was applied to a set of data acquired in the room described previously. The data acquisition took place during a system identification experiment where pseudo-random binary sequences were being applied to the air conditioning set-point, hence there was significant variability in the room environment. The result is shown in Fig. 6. The estimates provided by the model are extremely accurate: the average and maximum absolute errors were 0.0014 and 0.0075 .

A note is due on the important trade-off between computing time and estimation accuracy. Fig. 7 highlights the relative performance of RBF models in terms of computing time and accuracy. The lower plot shows the ratio between the PMV given by (1) and the one computed by an RBF ANN with $n$ neurons. It may be seen that a 13 neurons model is about 20 times faster than (1) and a 5 neurons model is approximately 55 times faster. The limit case of interest is 4 neurons corresponding to a speed-up near 70 .

The upper plot in the same figure shows the relative accuracy in terms of average absolute error. The limiting case of interest is, maybe, 12 neurons, about 20 times faster, with the double of the smaller average error achieved with 32 neurons. Although this plot may suggest that less than 12 neurons achieves a bad performance, the middle plot clears this impression. It shows the relative performance in terms of the maximum absolute error. It may be seen that with only 5 neurons the best performance is almost achieved, although in terms of average error the model is a bit worse than the best one. Using these curves one may select a specific model with the desired balance between accuracy and speed-up.

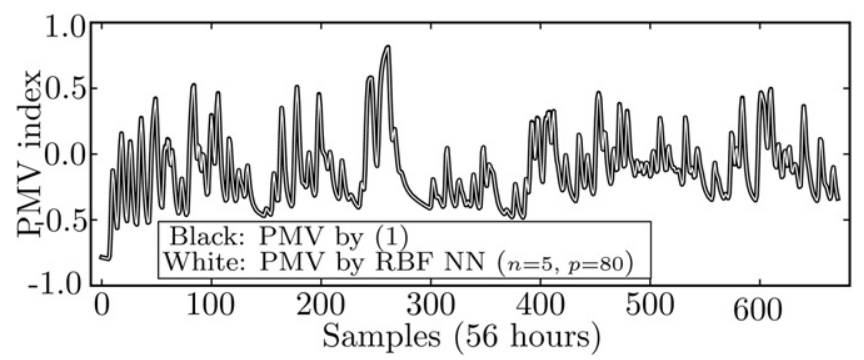

Fig. 6. PMV given by (1) (thick black line) and by 5 neuron RBF ANN (thin white line) during a system identification experiment. Please note that the lines are coincident, hence the white line is within the black line.

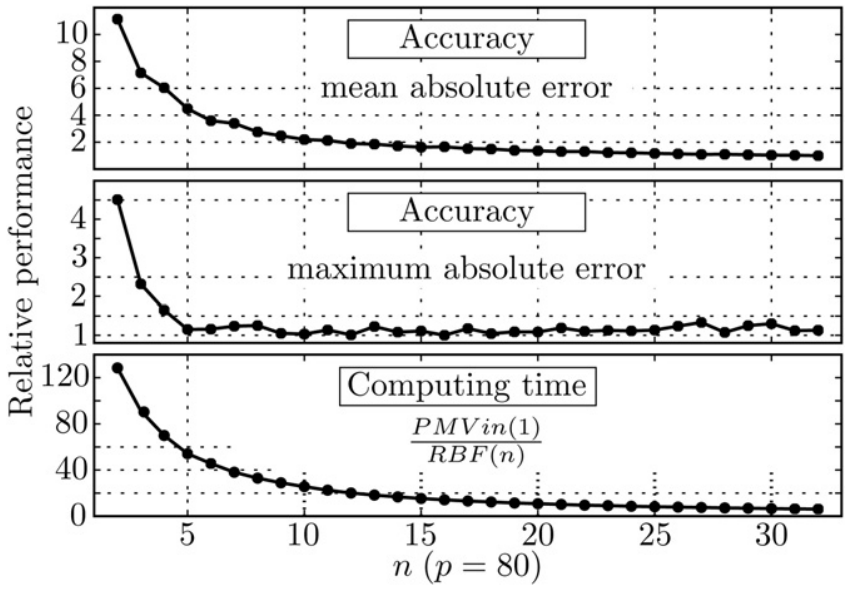

Fig. 7. Relative performance of the RBF PMV models regarding computing time and accuracy.

When compared to the models in [29] or [30], those here presented show better estimation accuracy, specifically on unseen data in real application, provide a wider coverage of the PMV input variables and of the thermal sensation scale, and achieve speed-up improvements. In this last case the gain is significant, being very large when compared to [30] and estimated to be about 3.5 times faster than the model in [29].

\section{Predictive models}

All the predictive models, needed in the MBPC, were implemented by RBF NNs, and were trained using the procedure described in Section 3.3. The topology of the model, i.e., its input-output structure and the number of neurons, was identified using a Multi-Objective Genetic Algorithm (MOGA). A complete description of the model structure identification procedure is beyond the scope of this paper, the interested reader should consult $[47,43,48,44]$.

Three auto-regressive predictive models were selected by MOGAs for $T_{a o}, H_{a o}$, and $R_{s g}$. They are used to forecast the outside weather and are employed whenever one of $T_{a o}, H_{a o}$, or $R_{s g}$ is necessary to obtain room temperature and humidity model predictions.

In order to obtain predictive air temperature and humidity models for a specific room, the first step was the preparation of control input signals for the HVAC internal unit. For that, the room was controlled randomly by varying the temperature set-point within the range $[18,19, \ldots, 27]$ or by switching off the unit for varying time intervals. This task was accomplished by means of Pseudo Random Binary Signals (PRBS), as described in [49,50]. Two sets of predictive air temperature and humidity models were designed, one to be used in winter conditions, and the other in summer. The following subsections describe their design.

\subsection{Summer models}

PRBS signals with 4416 data patterns were generated, corresponding to approximately 15 days of data at $5 \mathrm{~min}$ sampling interval. Different times-of-day were covered and distinct days (concerning the outside weather) were considered, all during early summer. Fig. 8 shows a sample PRBS sequence of set-points and the resulting room air temperature and relative humidity.

Using the MOGA and the techniques referenced above, predictive models for the room air temperature and humidity were selected. The first uses 14 neurons, the second uses 11 . The input variables and delays used by the selected models are detailed in Table 1 . As expected, there is some delay from the outside variables 

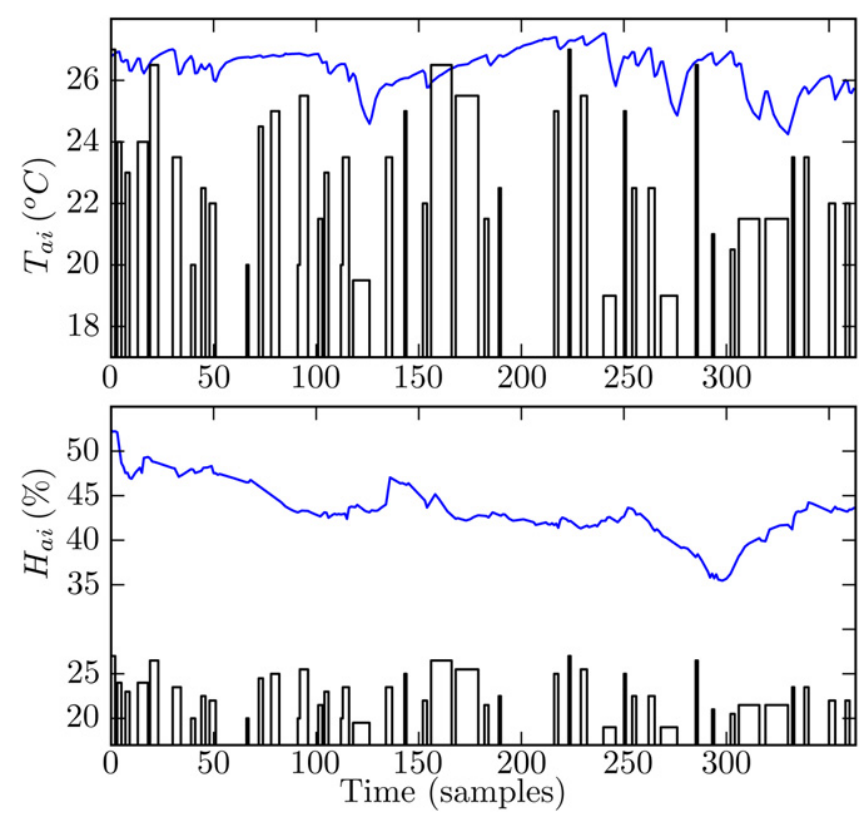

Fig. 8. Sample of PRBS sequence applied to HVAC system and the resulting temperatures and humidities. Summer conditions.

to the room climate models, as opposed to the inside variables and HVAC temperature set-point.

Within the model identification procedures the models were evaluated for long-term prediction capabilities using a subset of data. They were simulated for prediction over an horizon of 48 steps, which corresponds to $4 \mathrm{~h}$. Within this horizon the RMS error increased from about $0.06{ }^{\circ} \mathrm{C}$ up to approximately $0.65^{\circ} \mathrm{C}$ for air temperature, and from near $0.5 \%$ up to about $3.0 \%$ for relative humidity. These results were obtained by following the minimum error prediction approach, which means that measured values were used every time that future values of exogenous variables were necessary at the model inputs. Considering a $4 \mathrm{~h}$ prediction horizon, the error values may be considered quite small and adequate for the purpose of inclusion in a MBPC scheme.

\subsection{Winter models}

In this case, 2199 data patterns were generated, corresponding to approximately 8 days of data at 5 min sampling interval. Fig. 9 shows a sample of the PRBS sequence of temperature set-points and the resulting room air temperature and relative humidity.

Using the MOGA, predictive models for the room air temperature and humidity were selected. The first uses 7 neurons, the second uses 11 . The input variables and delays used by the selected models are presented in Table 2 .

As for summer models, the winter ones were also evaluated for long-term prediction, in this case within a prediction horizon of $2 \mathrm{~h}$ (24 steps-ahead). Within this horizon the RMS error increased from about $0.13^{\circ} \mathrm{C}$ up to approximately $0.26^{\circ} \mathrm{C}$ for air temperature, and from $0.5 \%$ up to near $2.5 \%$ for relative humidity. As with summer

Table 1

Input-output structure for room climate summer models.

\begin{tabular}{lll}
\hline Variable & Delays in $T_{a i}$ model & Delays in $H_{a i}$ model \\
\hline$T_{a i}$ & $0,1,6,7,8,10,11$ & $1,6,8$ \\
$H_{a i}$ & $0,1,2,7,9$ & $0,1,4,6,7,8,9,10$ \\
$T_{a o}$ & $2,3,4,5,8$ & - \\
$H_{a o}$ & - & $3,5,6,8,11$ \\
$R_{s g}$ & 7 & $6,8,11$ \\
$T_{s p}$ & $0,1,4$ & $0,1,2,3,6,9,10,11$ \\
\hline
\end{tabular}
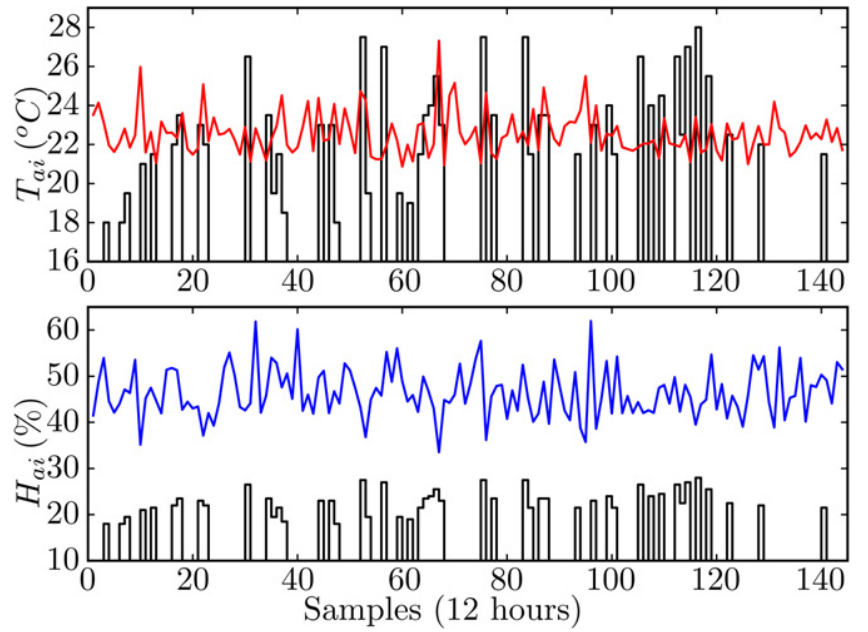

Fig. 9. $12 \mathrm{~h}$ sample of PRBS sequence of temperature set-points applied to the HVAC system and the resulting temperatures (top, red line) and humidities (bottom, blue line). Winter conditions. (For interpretation of the references to colour in this figure legend, the reader is referred to the web version of the article.)

models the minimum error approach was used, and the error values may also be considered quite small and amenable to be employed in a MBPC scheme.

\section{Discrete MBPC using branch and bound}

As mentioned in Section 1, when the control space is discrete or discretised it becomes possible to employ search techniques such as the BB method in order to find an optimal sequence of control actions that minimises a cost function. BB methods are structured search techniques commonly used to solve complex discrete optimisation and combinatorial programming problems by dividing them into smaller sub-problems using a tree structure. In a discrete MBPC formulation, the global problem is to find the optimal sequence of control actions over the prediction horizon. The choice of an adequate control action at every instant constitutes the various sub-problems to be solved. Assuming $A$ is a vector of $n$ possible control actions, at the initial step of the optimisation, in time instant $\mathrm{k}$, the BB method creates the initial tree node corresponding to the decision of what action should be taken at that time step. As $n$ control combinations are available, the corresponding number of branches is created by computing the predicted system output, $\hat{y}(k+1)$, and for each branch the cost function, $J(k+1)$, is evaluated. In the next prediction step, for $k+1$, the process is repeated for the nodes created in each branch resulting from the previous step, creating $n^{2}$ new branches. The whole process is repeated until time instant $k+P H-1$ is reached, where the number of created branches is $n^{P H}$. The exponential nature of the whole process is clear and even for a small number of control options and not too large prediction horizons, the number of available solutions quickly becomes prohibitively large. The optimal solution is chosen by selecting the control trajectory,

Table 2

Input-output structure for room climate winter models.

\begin{tabular}{cll}
\hline Variable & Delays in $T_{a i}$ model & Delays in $H_{a i}$ model \\
\hline$T_{a i}$ & $0,6,7,8,11$ & $1,5,8$ \\
$H_{a i}$ & $0,1,5,6,9,10,11$ & $0,1,3,6,8,10$ \\
$T_{a o}$ & 1,4 & - \\
$H_{a o}$ & - & $3,6,9,11$ \\
$R_{s g}$ & $0,3,4,8,9$ & $6,9,10$ \\
$T_{s p}$ & $0,1,3,10,11$ & $0,1,2,5,8,9,10,11$ \\
\hline
\end{tabular}


$\mathbf{U}(k)=[\mathbf{u}(k) \mathbf{u}(k+1) \cdots \mathbf{u}(k+P H-1)]$, that minimises the estimated accumulated cost from time instant $k+1$ to $k+P H$ :

$J_{1: P H}(k)=\sum_{i=k+1}^{k+P H} J(i)$

The description above assumes unrestricted branching and results in an enumerative search over the entire space of control solutions spanned by $A$ over the prediction horizon $P H$. As already pointed out this type of search easily becomes computationally prohibitive and in order to reduce the number of solutions enumerated, two approaches are taken: the use of bounds to restrict branching and performing the search over a control horizon, $\mathrm{CH}<\mathrm{PH}$. As formulated in [51], two bounds are employed: an upper bound on the total cost from instant $k+1$ to $k+P H$, and a lower bound on the cost from instant $k+i$ to $k+P H$. At time step $i$ in the optimisation a branch is followed only if the cumulative cost from step 1 to step $i-1, J_{1: i-1}(k)$, plus the lower bound on the cost from $i$ to $P H, \hat{J}_{i: P H}(k)$, is smaller than the upper bound on the total cost, $\hat{J}_{1: P H}(k)$. Thus the branching rule is given by:

$J_{1: i-1}(k)+\hat{J}_{i: P H}(k)<\hat{J}_{1: P H}(k)$

This rule may be further decomposed by noting that its second term on the left hand side of the condition equals the cost of using a control profile $A_{j}$ at step $i$,

$\left.J(k+i)\right|_{\mathbf{u}(k+i-1)=A_{j}}$,

plus the estimated cost from step $i+1$ to $P H$ :

$J_{1: i-1}(k)+\left.J(k+i)\right|_{\mathbf{u}(k+i-1)=A_{j}}+\hat{J}_{i+1: P H}(k)<\hat{J}_{1: P H}(k)$

When the rule does not hold the branch is not followed because it does not contain an optimal solution, thus pruning all the tree nodes that would be created from the current node. The bounds estimation method and availability are problem dependent, although a basic approach is suggested by [51]: at each instant $k$, before the optimisation starts, a first search on the tree of possible solutions is done by successively choosing the control action giving the smallest values of $J(k+i)_{i=1}^{P H}$, a search usually called "greedy". The total cost found is the initial estimated upper bound, $\hat{J}_{1: P H}(k)$. If at a later stage in the optimisation a smaller value is found, it replaces the previous one. Regarding the lower bound, $\hat{J}_{i+1: P H}(k)$, if an adequate estimate may not be computed, it is suggested that it is set to 0 for all steps $i$ of the optimisation. In the typical formulation, branching is only performed until the control horizon is reached, therefore the remaining cost must be estimated for all instants from $\mathrm{CH}+1$ up to $\mathrm{PH}$, for example by using the greedy approach just described. It is worth noting some advantages of the BB method over other non-linear optimisation techniques when applied to MBPC:

- The optimal solution is always found. This guarantees that the controller is optimal in the discrete space of control alternatives. Importantly, no assumptions need to be made on the formulation of the cost function.

- The method implicitly deals with constraints without being negatively affected. Constraints will most certainly improve the efficiency of bounding by eliminating those alternatives that lead to constraint violation.

- As opposed to other iterative optimisation methods, the algorithm outcome is not negatively influenced by a poor initialisation, although the time spent to find the optimum may be greater.

\section{Problem formulation}

In order to maintain thermal comfort and simultaneously minimise the energy spent, the problem may be formulated as follows. The cost of selecting one control action, $T_{s p}$, at instant $i$ is defined as,

$\hat{J}(i)= \begin{cases}1+\frac{\left|T_{s p}-T_{a o}\right|}{\lambda}, & T_{s p}>0 \\ 0, & T_{s p}=0\end{cases}$

where $T_{s p}=0$ encodes the action of switching off the HVAC unit. The $\lambda$ scaling factor is used only to make that term small when compared to 1 . In practice it should be chosen by taking into account an estimate of the maximum value of $\left|T_{s p}-T_{a o}\right|$. The term itself reflects the notion that the higher the difference $\left|T_{s p}-T_{a o}\right|$, the bigger is the energy required to achieve $T_{s p}$. Using the definition (17) the HVAC control problem is simply,

$$
\begin{array}{ll}
\underset{\mathbf{U}(k)}{\operatorname{minimise}} & \mathcal{J} 1: P H(k)=\left.\left(\sum_{i=k+1}^{k+P H} \hat{J}(i)\right)\right|_{\mathbf{U}(k)}, \\
\text { subject to } & |\hat{\Theta}(i)|<\Theta_{T}
\end{array}
$$

where $\hat{\Theta}(i)$ is the estimated PMV index resulting from selecting the set-point $T_{s p}$ at time instant $i$. $\Theta_{T}$ is a threshold value for the PMV index which should guarantee acceptable thermal comfort for the occupants of the space. The ASHRAE standard [26] recommends a value of 0.5 which predicts that less than $10 \%$ of the occupants will be dissatisfied.

\section{Results of the MBPC approach}

Using the methodologies described in previous sections, a number of experiments, both in summer and winter periods, have been carried out to test the functionality and assess the correctness and robustness of the control system.

\subsection{Summer experiments}

The results that will be presented and discussed in this section were obtained in a classroom equipped with computers, where students have a number of courses on different computer science topics (room D described in Section 2). After making a number of systematic measurements on the air velocity within the room for different settings of the HVAC fan speed, it was concluded that, excluding the vicinity of the air ducts, the velocity was on average below $0.1 \mathrm{~ms}^{-1}$. When computing the PMV index, a value of $0.65 \mathrm{clo}$ was used for the clothing insulation and a value of 1.0 Met was employed for the metabolic rate. This means that the context, for the PMV models used, was $C=\{0.65,1.0,0.1\}$. Regarding the MBPC system parameters, the control horizon, $\mathrm{CH}$, was set to 5 samples ( $25 \mathrm{~min}$ ) and the prediction horizon, $\mathrm{PH}$, to 48 samples ( $4 \mathrm{~h}$ ).

\subsubsection{Experiment 1}

Fig. 10 presents one situation of a hot summer day where the room was in use when the system started operation. The initial thermal comfort index, $\Theta$, is above the 0.5 threshold and the HVAC takes almost $2 \mathrm{~h}$ at $18{ }^{\circ} \mathrm{C}$ set-point to bring the room to acceptable thermal comfort conditions. Beyond this point, with the room with no load, the system is able to maintain the desired conditions by using higher set-points and by switching off the HVAC when possible, therefore consuming less energy.

The room air temperature model does not have an input accounting for the room usage, consequently the control algorithm is not able to foresee and act pre-emptively to counteract the strong impact caused by a class entering the room. 


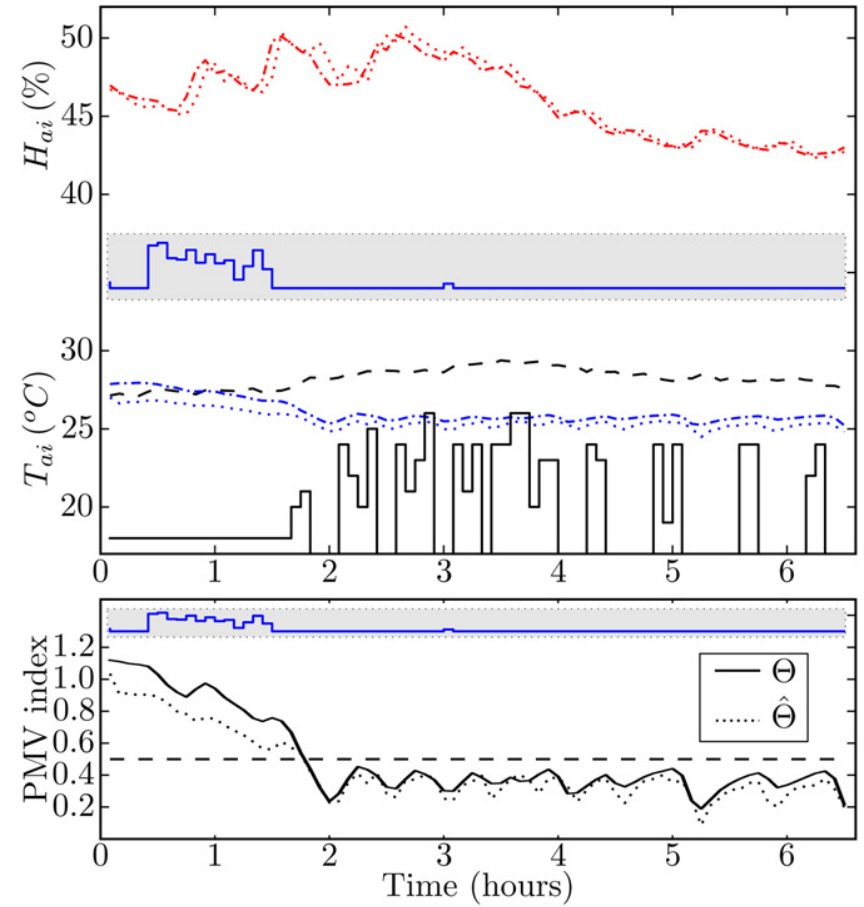

Fig. 10. HVAC control for thermal comfort in summer conditions. Room is in use when system starts. Both plots: shaded area shows room activity monitor signal. Upper plot: measured (red dash-dot) and one-step-ahead predicted (red dot) humidity, are shown above shaded area; same (in blue) below shaded area for air temperature; dash and solid lines show outside air temperature and AC set-point. (For interpretation of the references to colour in this figure legend, the reader is referred to the web version of the article.)

\subsubsection{Experiment 2}

Fig. 11 presents a second situation, on an even slightly hotter day, where after a first time span of room occupancy ending with acceptable thermal conditions, the system was able to maintain those conditions on a second class with less effort than that of the first case. This would be the expected behaviour if the system could predict the room occupancy.

\subsubsection{Experiment 3}

On what concerns the room air temperature and relative humidity models, it may be seen that the predictions are quite accurate which results on a good forecast of the PMV index and correct operation of the system through time. This is confirmed by the results presented in Fig. 12, which give good indications regarding robustness. The system operated for about $48 \mathrm{~h}$ maintaining good thermal conditions and showing excellent room climate modelling, also accounting positively on the outside weather models. If they would not provide sufficiently accurate predictions the room climate models accuracy would suffer.

\subsection{Winter experiments}

The aim of the following experiments was not only to test the functionality and assess the correctness and robustness of the control system, in winter conditions, but also to assess any possible energy savings that it may generate. In order to fulfil the second goal, 3 adjacent similar rooms were used. The objective is to run the MBPC algorithm in one of the rooms and compare the results to those obtained by the HVAC built-in control system in the other rooms. Please recall the rooms description that was given in Section 2. Room $A$ and $C$ will employ the conventional control system, and MBPC will be applied in Room B. After making a number of
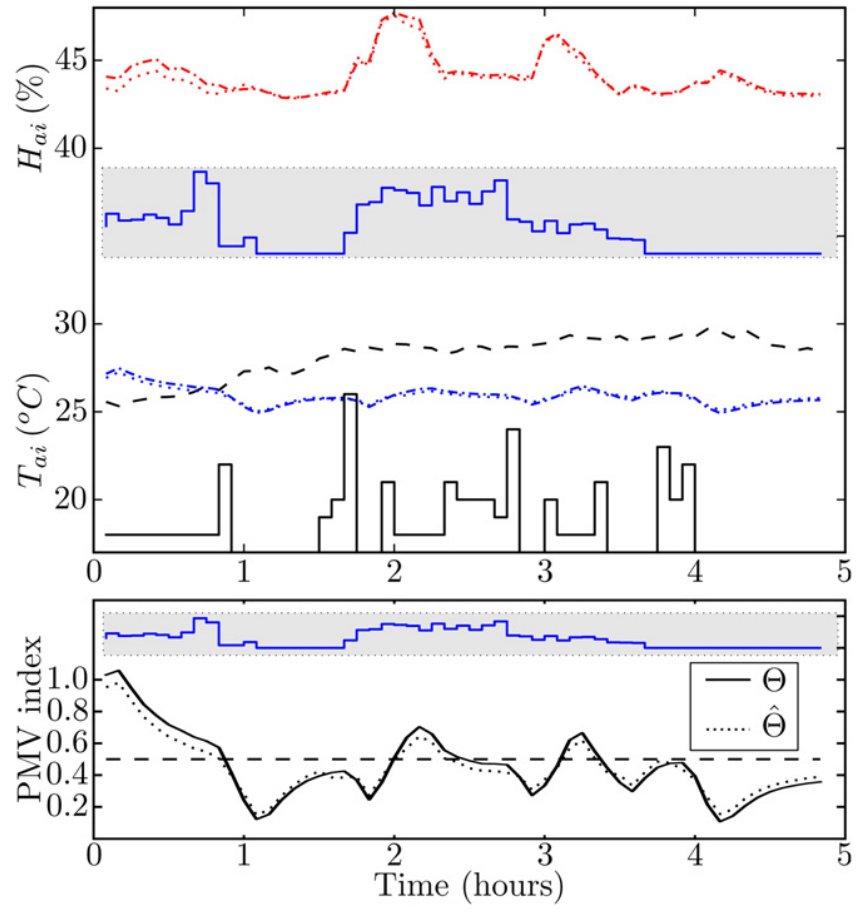

Fig. 11. HVAC control for thermal comfort in summer conditions. Pre-emptive control improves performance. The same structure, line style and colour coding of Fig. 10 was used. (For interpretation of the references to colour in this figure legend, the reader is referred to the web version of the article.)

systematic measurements on the air velocity within the 3 rooms for different settings of the HVAC fan speed, it was concluded that, excluding the vicinity of the air ducts, the velocity was on average close to $0.08 \mathrm{~ms}^{-1}$. A value of 1.0 clo was used for the clothing insulation and a value of 1.0 Met for the metabolic rate. A PMV model with context $C=\{1.0,1.0,0.08\}$ was therefore employed. Regarding the MBPC system parameters, the control horizon, $\mathrm{CH}$, was again set to 5 samples ( $25 \mathrm{~min}$ ) and the prediction horizon to 48 samples (4h).

\subsubsection{Experiment 4}

Fig. 13 shows the results of a first small experiment carried out to test the experimental set-up.

In room B (solid red line) the set-point is determined by the MBPC system. In rooms A (black dash-dot line) and C (blue dash line), fixed set-points of 23 and $22^{\circ} \mathrm{C}$ were employed, respectively. These values are commonly used as pre-programmed set-points for the rooms within the HVAC management system. The occupants frequently set even larger values. The experiment took place at the end of the day, during which the average outside air temperature was $8.8^{\circ} \mathrm{C}$.

It may be seen that initially all the rooms are outside the comfort zone as the PMV index is below -0.5 . After about $20 \mathrm{~min}$ all the rooms were within the comfort zone, in the case of B by action of the MBPC system which shows good responsiveness to the vicinity of the PMV index threshold of -0.5 . Whenever the system foresees that the threshold will be reached it determines one of the possible set-points and applies it to the system. This is an indication of the good predictive ability of the models and of their usefulness for the BB solution search. It is also obvious that the HVAC system temperature regulation is very poor, as in rooms $A$ and $C$ the temperature was always far away from the target value. The result is that the system was permanently heating for approximately $2 \mathrm{~h}$ and 20 min. In room $B$ the MBPC was making good decisions as it 


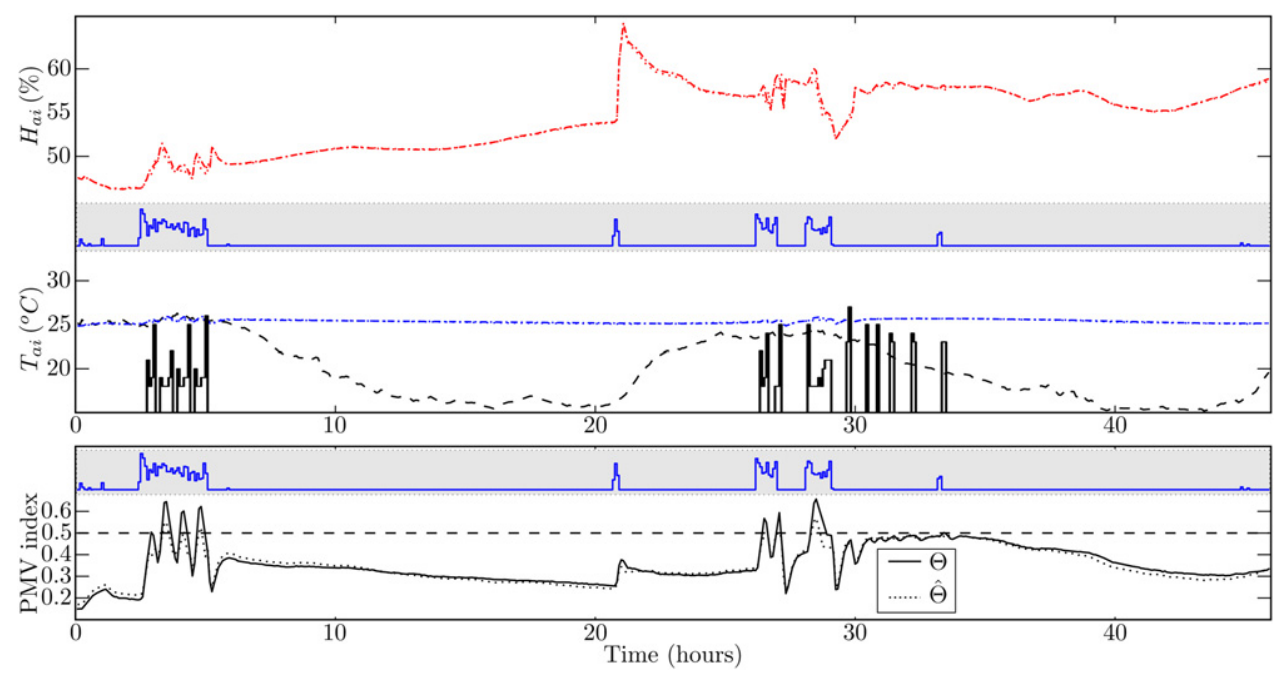

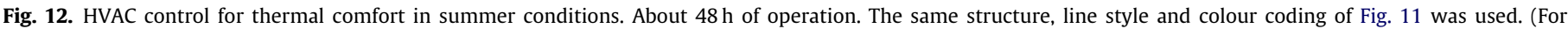
interpretation of the references to colour in this figure legend, the reader is referred to the web version of the article.)

was maintaining the comfort index above the -0.5 limit without switching on the AC permanently. During the $2: 20 \mathrm{~h}$ that the AC was heating in rooms $A$ and $C$, the total active time in room $B$ was $55 \mathrm{~min}$, which corresponds to a reduction of about $60 \%$.

\subsubsection{Experiment 5}

A second experiment was conducted in order to verify the results obtained in the first short experiment. It started early in the day, around 6:00 a.m. and lasted until 12:30. During this period the outside air temperature increased from 7.5 to $16.5^{\circ} \mathrm{C}$, with an average of 11.7. The settings and set-points were the same as for the first experiment. The results are illustrated in Fig. 14 using the same line style and colour coding as in Fig. 13.

During the first $4 \mathrm{~h}$ of this experiment the behaviour was similar to the one observed in the first experiment. At that point the system in room $C$ started to switch on and off the AC system as if it had reached the set-point, although, as measured by the WSN, it did not. Surprisingly, as it seemed to have reached its set-point, up to the end of the experiment the AC system kept increasing the temperature in that room. In room A, the same problems as before were observed, as the AC system is continuously active.

The MBPC system in room B managed very well to maintain the thermal comfort and have relatively large periods of inactivity. When compared to rooms $\mathrm{A}$ and $\mathrm{C}$ the reduction in heating time was approximately $41 \%$ and $45 \%$, respectively. The results achieved reinforce the idea that the existing HVAC system provides inadequate temperature regulation and has quite different performance among the rooms. Because of this an additional experiment was carried out only in room B.

In order to be able to compare the MBPC in room B with the standard control algorithm in the same room, right after the second experiment the MBPC was substituted by a fixed set-point of $22^{\circ} \mathrm{C}$.
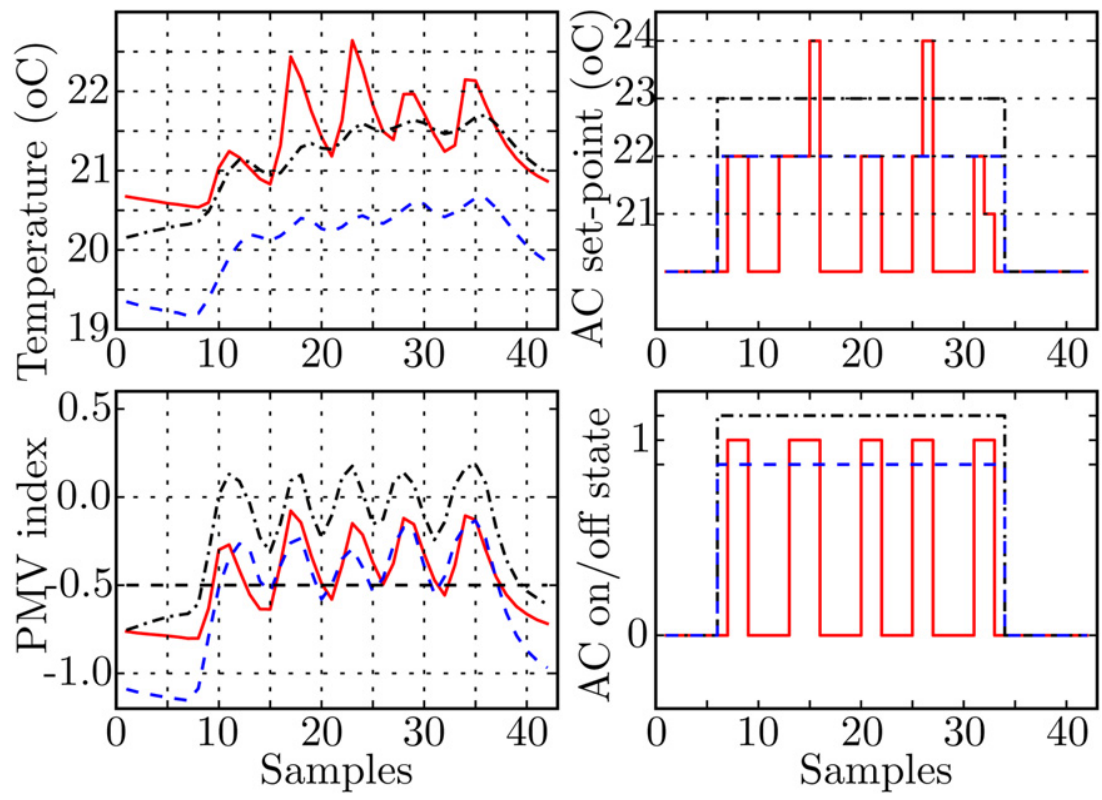

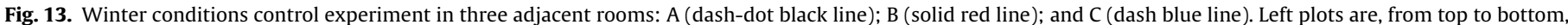

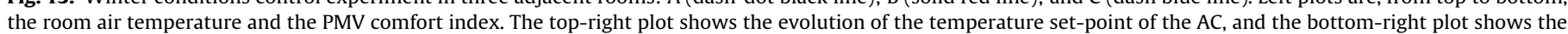
state, on or off, of the AC. (For interpretation of the references to colour in this figure legend, the reader is referred to the web version of the article.) 

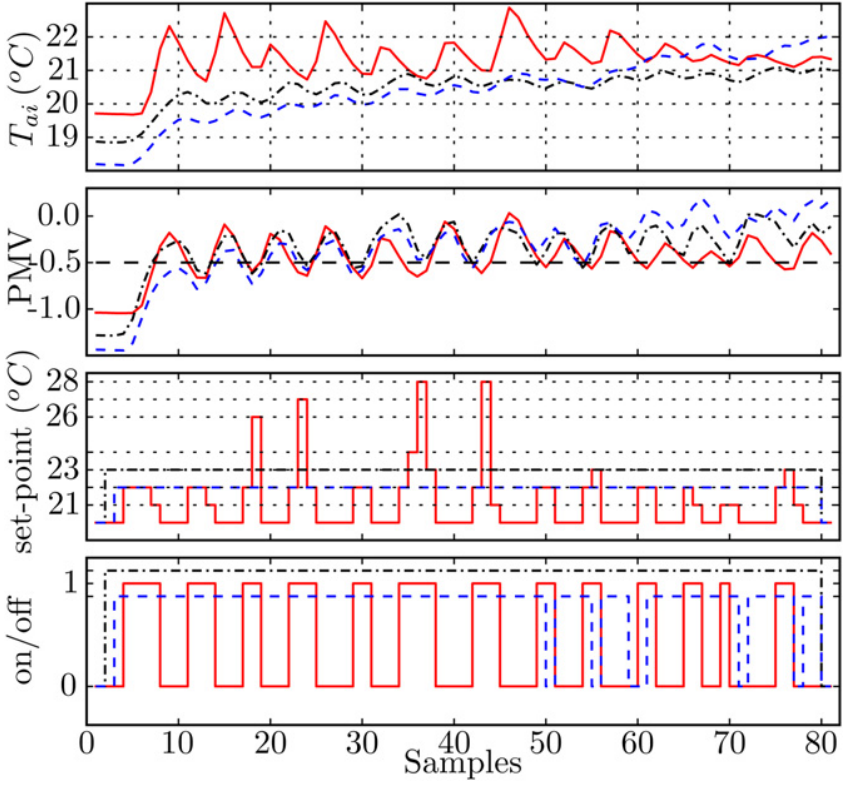

Fig. 14. A second experiment to estimate relative energy savings in winter conditions. The same colour and line type coding as in Fig. 13, was used for rooms A, B, and $C$. (For interpretation of the references to colour in this figure legend, the reader is referred to the web version of the article.)

This set-point results in a PMV index value not far away from -0.5 , the threshold that was governing the behaviour of the MBPC. As the outside temperature was still rising, the fixed set-point control task would not be harder when compared to the previous couple of hours in the second experiment where the outside temperatures were smaller, therefore to a good extent the last $2 \mathrm{~h}$ of the second experiment are comparable to the first couple of hours of the fixed set-point experience, without favouring the MBPC.

The results from the comparison are visible in Fig. 15. As in previous experiments the MBPC keeps good track of the thermal comfort restriction which is only marginally violated because of the inevitable error of predictive models. The system only spends energy when necessary to maintain the minimum level of comfort specified. Regarding the standard temperature regulation (in dash-dot black line), it may be seen that regardless of attaining the set-point and of switching on and off some times, it keeps increasing the room temperature unnecessarily, therefore wasting energy. In this comparison the MBPC activated the AC system approximately $37 \%$ less than the HVAC control.

\subsubsection{Experiment 6}

On what concerns the room air temperature, relative humidity and PMV index, it may be seen in Fig. 16 that the one-step-ahead predictions are accurate, resulting on good PMV index forecasts and on correct operation of the system through time, which give good indications regarding robustness. This figure was obtained by letting the system run for approximately $11 \mathrm{~h}$, where the unit was heating only $15 \%$ of this period. During this time the system operated correctly and continuously, always maintaining desired thermal conditions and showing excellent room climate modelling, also accounting positively on the outside weather models.

It should be noted that from sample 40 up to sample 95 , the room was being used for lectures. This represents a thermal load that actually contributes to maintain the comfort during winter. On summer conditions, as it was seen, the system is required to react, therefore, as in summer models, an input must be added to the models to account for the room occupancy schedule.
Table 3

Energy (in kWh) assigned to each indoor unit.

\begin{tabular}{lrlrr}
\hline Experiment & \multicolumn{1}{c}{$\mathrm{A}$} & $\mathrm{B}$ & \multicolumn{1}{c}{$\mathrm{C}_{1}$} & \multicolumn{1}{c}{$\mathrm{C}_{2}$} \\
\hline 4 & 5.3 & 2.5 & 5.3 & 4.4 \\
5 & 13.6 & 6.9 & 14.6 & 11.8 \\
6 & 24.8 & 5.6 & 24.8 & 16.3 \\
\hline
\end{tabular}

\subsection{Energy savings}

As within the 16 locations monitored there are 14 interior air conditioning units, it was not economically possible to actually measure the electric energy spent in every indoor unit. As a compromise solution, power transducers were installed in the three outdoor units, therefore making the electric energy values available for these units through the LMAP02 interface. A procedure, suggested by Mitsubishi, was then followed to give a first, crude approximation to the electric energy consumption of each indoor unit [52].

Denoting by $E_{0}(T)$ the electrical energy consumed by the outdoor unit, during a period $T$, by $T_{\text {oni }}$ and $T_{\text {thi }}$ the time (in hours) that the fan is operating of the $i$ th indoor unit and that the unit is cooling or heating, respectively, then the energy $E_{i}(T)$ (in Wh), assigned to the ith indoor unit, out of $N$ units, is given by:

$E_{i}(T)=E_{o}(T) \frac{T_{\text {thi }}}{\sum_{i} T_{\text {thi }}}+220 T_{\text {on } i}+\frac{32}{N}$

The constants in (19) have been computed for the models in use, following [52].

In experiments 4-6, the indoor units of rooms $A, B$ and $C$ were connected to the same outdoor unit. Room $A$ and $B$ have a single indoor unit while room $B$ had 2 . Rooms $A$ and $C$ were under constant temperature control, whilst in room B the proposed MBPC methodology was controlling the indoor unit. The rooms were adjacent, located in the left corner of the building (please see Fig. 1). The area of room $A$ is equal to the area of room $B$ and half of room $C$ (hence the two indoor units). The total electric energy (in kWh) assigned to each unit, for each experiment, and using the procedure described above, is given in Table 3.

Table 4 shows the savings, in percentage, achieved in the electric energy spent by unit B, compared with all the other units, for each experiment. As it can be seen, the savings range from $41 \%$, achieved in the 5th experiment and taking the 2nd interior unit of room $C$ as the comparison unit, and $77 \%$, in experiment 6 , for the interior unit in room $A$ and the 1 st interior unit of room $C$. These values of energy savings, if on one hand were expected because the system has the ability to use future information and because it uses more and diverse information, on the other hand they also occur due to the inadequate operation of the standard control in the HVAC system. This may occur for various reasons: malfunctions, inadequate sensor locations, bad quality equipment, excessively simple control algorithms, or inadequate set-points being set. Whatever the reason is, the results obtained show that by having a MBPC commanding the HVAC system, important savings in energy, in the order of $50 \%$, are to be expected.

Table 4

Savings (in \%) of B compared with $\mathrm{A}, \mathrm{C}_{1}$ and $\mathrm{C}_{2}$.

\begin{tabular}{llll}
\hline Experiment & $\mathrm{A}$ & $\mathrm{C}_{1}$ & $\mathrm{C}_{2}$ \\
\hline 4 & 52 & 52 & 43 \\
5 & 49 & 52 & 41 \\
6 & 77 & 77 & 66 \\
\hline
\end{tabular}



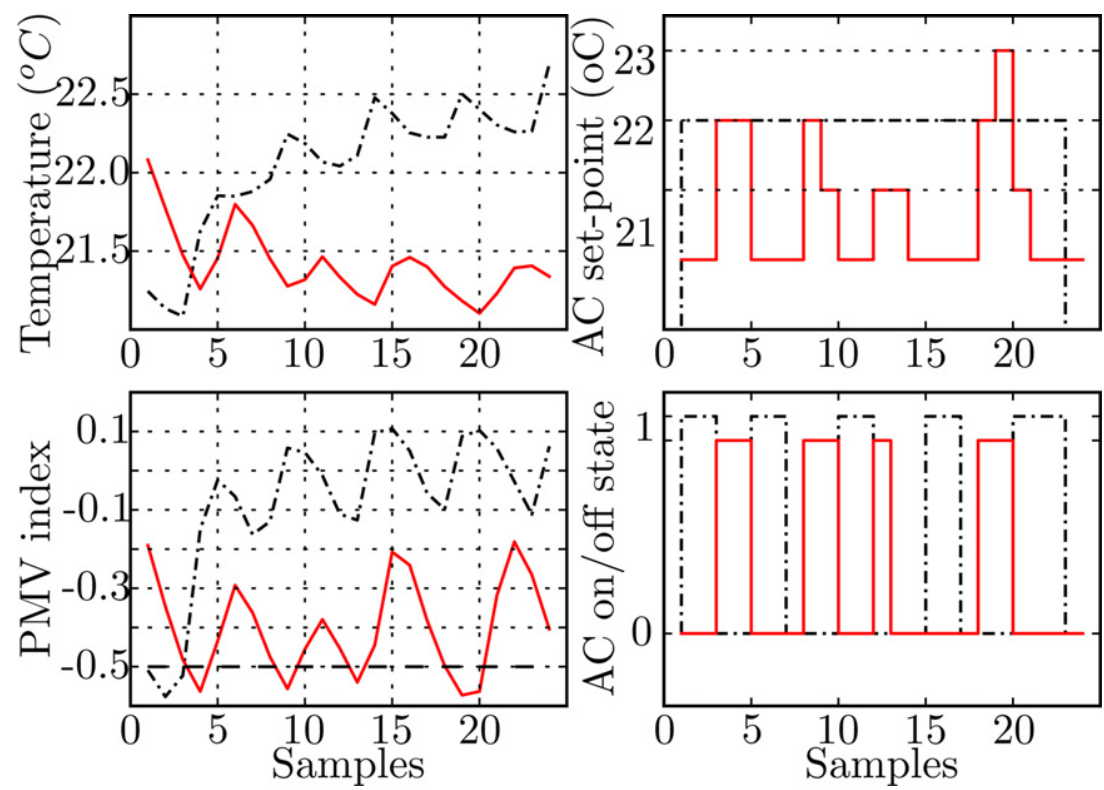

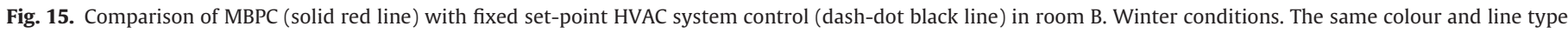
coding as in Fig. 14, was used for room B. (For interpretation of the references to colour in this figure legend, the reader is referred to the web version of the article.)
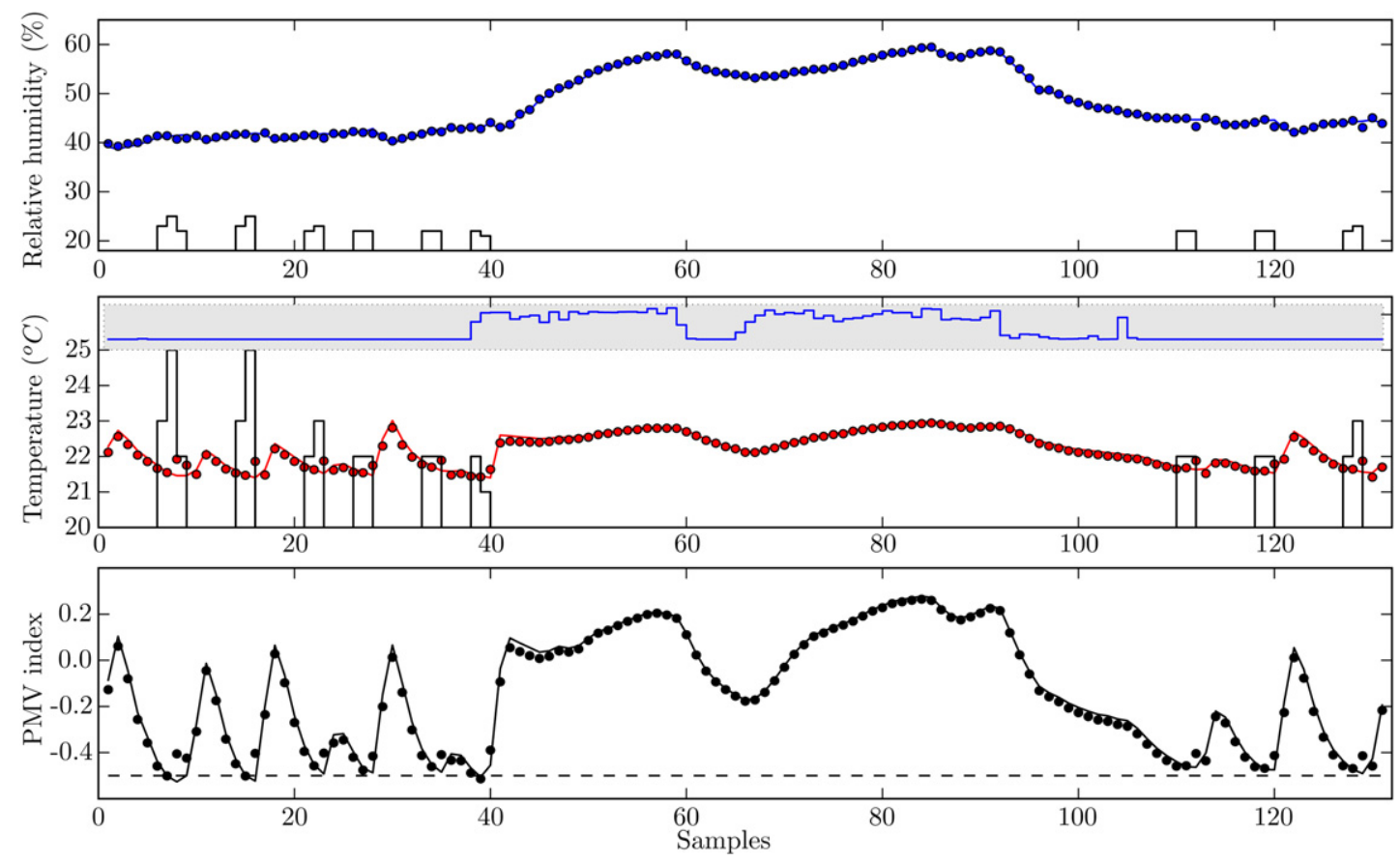

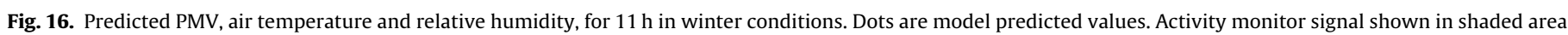
of middle plot.

\section{Conclusions and future work}

A model based predictive control methodology using the branch and bound method was formulated and applied to control existing HVAC systems in buildings. To the extent the models accuracy allow, the formulation guarantees that an optimal control trajectory is computed in order to maintain a desired level of thermal comfort and to minimise the energy spent in doing so. The system is conceptually simple and integrates easily with existing HVAC systems. The feasibility and robustness have been demonstrated experimentally. According to the results, important energy savings are achieved by having the model based predictive controller determining the operation of HVAC systems. These savings are probably above $50 \%$.

As the model based predictive control needs several calculation of the predicted mean value index, its computation, using a set of radial basis function neural network models, has been investigated. The models show good estimation accuracy over wide ranges of the input variables and provide good coverage of the thermal sensation scale. When compared to previous works, the design of the models was planned in detail with the purpose of using them in real-time control applications, the generalisation of the models was 
tested thoroughly, and a procedure was shown in order to select a model on the basis of a desired compromise between speed-up and estimation.

Future work will focus on the improvement of the control system by making it aware of the rooms occupancy schedule, and on estimating more accurately the potential energy savings.

\section{Acknowledgements}

The authors wish to acknowledge the support of the Portuguese National Science Foundation (project grant PTDC/ENR/73345/2006), the European Commission for the grant PERG-GA-2008-239451 and the University of Algarve for the Ceratonia 2008 Award. This work was also supported by FCT, through IDMEC, under LAETA.

\section{References}

[1] B. Poel, G. van Cruchten, C.A. Balaras, Energy performance assessment of existing dwellings, Energy and Buildings 39 (2007) 393-403, http://dx.doi.org/10.1016/j.enbuild.2006.08.008.

[2] C. Balaras, E. Dascalaki, A. Gaglia, K. Droutsa, S. Kontoyiannidis, Energy performance of European buildings, in: Proceedings of the Energy Sustainability Conference, Long beach, USA, 2007, pp. 387-396.

[3] E.U. Parliament, E.U. Council, On the Energy Performance of Buildings, 2010, June. http://dx.doi.org/10.3000/17252555.L_2010.153.eng

[4] A. Dexter, Intelligent buildings: fact or fiction? HVAC\&R Research 2 (2) (1996) 105-106, http://dx.doi.org/10.1080/10789669.1996.10391336.

[5] A.E. Ben-Nakhi, M.A. Mahmoud, Energy conservation in buildings through efficient A/C control using neural networks, Applied Energy 73 (1) (2002) 5-23, http://dx.doi.org/10.1016/S0306-2619(02)00027-2.

[6] S. Wang, Y. Chen, Fault-tolerant control for outdoor ventilation air flow rate in buildings based on neural network, Building and Environment 37 (7) (2002) 691-704, http://dx.doi.org/10.1016/S0360-1323(01)00076-2.

[7] A.E. Ben-Nakhi, M.A. Mahmoud, Cooling load prediction for buildings using general regression neural networks, Energy Conversion and Management 45 (13-14) (2004) 2127-2141, http://dx.doi.org/10.1016/j.enconman.2003.10.009.

[8] S. Wang, Z. Ma, Supervisory and optimal control of building HVAC systems: a review, HVAC\&R Research 14 (1) (2008) 3-32, http://dx.doi.org/10.1080/10789669.2008.10390991.

[9] A. Dounis, C. Caraiscos, Advanced control systems engineering for energy and comfort management in a building environment - a review, Renewable and Sustainable Energy Reviews 13 (6-7) (2009) 1246-1261, http://dx.doi.org/10.1016/j.rser.2008.09.015.

[10] C. Chang, J. Zhao, N. Zhu, Energy saving effect prediction and post evaluation of air-conditioning system in public buildings, Energy and Buildings 43 (11) (2011) 3243-3249, http://dx.doi.org/10.1016/j.enbuild.2011.08.025.

[11] S. Soyguder, Intelligent system based on wavelet decomposition and neural network for predicting of fan speed for energy saving in HVAC system, Energy and Buildings 43 (4) (2011) 814-822, http://dx.doi.org/10.1016/j.enbuild.2010.12.001.

[12] T. Olofsson, S. Andersson, R. Östin, A method for predicting the annual building heating demand based on limited performance data, Energy and Buildings 28 (1) (1998) 101-108, http://dx.doi.org/10.1016/S0378-7788(98)00004-8.

[13] B. Dong, C. Cao, S.E. Lee, Applying support vector machines to predict building energy consumption in tropical region, Energy and Buildings 37 (5) (2005) 545-553, http://dx.doi.org/10.1016/j.enbuild.2004.09.009.

[14] P.A. González, J.M. Zamarre no, Prediction of hourly energy consumption in buildings based on a feedback artificial neural network, Energy and Buildings 37 (6) (2005) 595-601, http://dx.doi.org/10.1016/j.enbuild.2004.09.006.

[15] J. Yang, H. Rivard, R. Zmeureanu, On-line building energy prediction using adaptive artificial neural networks, Energy and Buildings 37 (12) (2005) 1250-1259, http://dx.doi.org/10.1016/j.enbuild.2005.02.005.

[16] S. Karatasou, M. Santamouris, V. Geros, Modeling and predicting building's energy use with artificial neural networks: methods and results, Energy and Buildings 38 (8) (2006) 949-958, http://dx.doi.org/10.1016/j.enbuild.2005.11.005.

[17] A.H. Neto, F.A.S. Fiorelli, Comparison between detailed model simulation and artificial neural network for forecasting building energy consumption, Energy and Buildings 40 (12) (2008) 2169-2176, http://dx.doi.org/10.1016/j.enbuild.2008.06.013.

[18] K. Li, H. Su, Forecasting building energy consumption with hybrid genetic algorithm-hierarchical adaptive network-based fuzzy inference system, Energy and Buildings 42 (11) (2010) 2070-2076, http://dx.doi.org/10.1016/j.enbuild.2010.06.016.

[19] G. Escrivá-Escrivá, C. Álvarez-Bel, C. Roldán-Blay, M. Alcázar-Ortega, New artificial neural network prediction method for electrical consumption forecasting based on building end-uses, Energy and Buildings 43 (11) (2011) 3112-3119, http://dx.doi.org/10.1016/j.enbuild.2011.08.008.
[20] K. Li, H. Su, J. Chu, Forecasting building energy consumption using neural networks and hybrid neuro-fuzzy system: a comparative study, Energy and Buildings 43 (10) (2011) 2893-2899, http://dx.doi.org/10.1016/j.enbuild.2011.07.010.

[21] A. Ruano, E. Crispim, E. Conceiç ao, M. Lúcio, Prediction of building's temperature using neural networks models, Energy and Buildings 38 (6) (2006) 682-694, http://dx.doi.org/10.1016/j.enbuild.2005.09.007.

[22] A. Ruano, E. Crispim, P. Fraz a, MOGA design of neural network predictors of inside temperature in public buildings, in: V. Balas, J. Fodor, A. Várkonyi-Kóczy (Eds.), Soft Computing Based Modeling in Intelligent Systems, vol. 196 of Studies in Computational Intelligence, Springer, Berlin/Heidelberg, 2009, pp. 35-61, http://dx.doi.org/10.1007/978-3-642-00448-3_3

[23] Y. Tachwali, H. Refai, J. Fagan, Minimizing HVAC energy consumption using a wireless sensor network, in: Industrial Electronics Society, 2007. IECON 2007. 33rd Annual Conference of the IEEE, 2007, pp. 439-444, http://dx.doi.org/10.1109/IECON.2007.4460329.

[24] D.-M. Han, J.-H. Lim, Design and implementation of smart home energy management systems based on zigbee, IEEE Transactions on Consumer Electronics 56 (3) (2010) 1417-1425, http://dx.doi.org/10.1109/TCE.2010. 5606278.

[25] T. Bedford, C. Warner, The globe thermometer in studies of heating and ventilation, Journal of Hygiene 34 (4) (1934) 458-473.

[26] M.S. Owen (Ed.), 2009 ASHRAE Handbook - Fundamentals, SI edition, ASHRAE, 2009.

[27] TinyOs, TinyOs Operating System, 1999, http://www.tinyos.net/

[28] Mitsubishi Electric, Air Conditioner Interface. Model LMAP02. Installation Manual, 2001, http://www.tinyos.net/

[29] S. Atthajariyakul, T. Leephakpreeda, Neural computing thermal comfort index for HVAC systems, Energy Conversion and Management 46 (15-16) (2005) 2553-2565, http://dx.doi.org/10.1016/j.enconman.2004.12.007.

[30] M. Kumar, I. Kar, Non-linear HVAC computations using least square support vector machines, Energy Conversion and Management 50 (2009) 1411-1418, http://dx.doi.org/10.1016/j.enconman.2009.03.009.

[31] Y. Jian, X. Jin, Research on the BPNN in the prediction of PMV Applied Mechanics and Materials 29-32 (2010) 2804-2808, http://dx.doi.org/10.4028/www.scientific.net/AMM.29-32.2804.

[32] ANSI, ASHRAE, Thermal Environmental Conditions for Human Occupancy, 2004.

[33] P.O. Fanger, Thermal Comfort: Analysis and Applications in Environmental Engineering, McGraw-Hill, New York, 1972.

[34] J. Kang, Y. Kim, H. Kim, J. Jeong, S. Park, Comfort sensing system for indoor environment, in: International Conference on Solid State Sensors and Actuators, vol. 1, 1997, pp. 311-314, http://dx.doi.org/10.1109/SENSOR.1997. 613646.

[35] I.O. for Standardization (ISO), ISO 7730: Moderate Thermal Environments Determination of the PMV and PPD Indices and Specification of the Conditions for Thermal Comfort, 1994

[36] K. Cena, J. Clark (Eds.), Bioengineering, Thermal Physiology and Comfort, vol. 10 of Studies in Environmental Science, Elsevier B.V., 1981.

[37] K. Levenberg, A method for the solution of certain non-linear problems in least squares, The Quarterly of Applied Mathematics (2) (1944) 164-168.

[38] D.W. Marquardt, An algorithm for least-squares estimation of nonlinear parameters, Journal of the Society for Industrial and Applied Mathematics 11 (2) (1963) 431-441.

[39] A. Ruano, D. Jones, P. Fleming, A new formulation of the learning problem of a neural network controller, in: Proceedings of the 30th IEEE Conference on Decision and Control, vol. 1, 1991, pp. 865-866, http://dx.doi.org/10.1109/CDC.1991.261439.

[40] P.M. Ferreira, A.E. Ruano, Exploiting the separability of linear and non-linear parameters in radial basis function neural networks, in: IEEE Symposium 2000: Adaptive Systems for Signal Processing, Communications, and Control, Canada, 2000, pp. 321-326, http://dx.doi.org/10.1109/ASSPCC.2000.882493.

[41] S. Haykin, Neural Networks: A Comprehensive Foundation, 2nd ed., Prentice Hall, Inc., 1999.

[42] P.M. Ferreira, E. Faria, A.E. Ruano, Neural network models in greenhouse air temperature prediction, Neurocomputing 43 (1-4) (2002) 51-75, http://dx.doi.org/10.1016/S0925-2312(01)00620-8.

[43] A.E. Ruano, P.M. Ferreira, C. Fonseca, An overview of non-linear identification and control with neural networks, in: A.E. Ruano (Ed.), Intelligent Control Using Soft-Computing Methodologies, vol. 70 of Control Series, IEE Publishing, 2005, pp. 37-87.

[44] P.M. Ferreira, A.E. Ruano, Evolutionary multiobjective neural network models identification: evolving task-optimised models, in: New Advances in Intelligent Signal Processing, vol. 372/2011 of Studies in Computational Intelligence, Springer, 2011, pp. 21-53, http://dx.doi.org/10.1007/978-3-64211739-8_2.

[45] J. Sjöberg, L. Ljung, Overtraining, regularization, and searching for minimum with application to neural networks, in: Preprint IFAC Symposium on Adaptive Systems in Control and Signal Processing, 1994, pp. 669-674.

[46] S. Amari, N. Murata, K.-R. Müller, M. Finke, H. Yang, Asymptotic statistical theory of overtraining and cross-validation, IEEE Transactions on Neural Networks 8 (5) (1997) 985-996, http://dx.doi.org/10.1109/72.623200.

[47] P.M. Ferreira, A.E. Ruano, C. Fonseca, Genetic assisted selection of RBF model structures for greenhouse inside air temperature prediction, in: IEEE Conference on Control Applications, Istanbul, Turkey, 2003, pp. 576-581, http://dx.doi.org/10.1109/CCA.2003.1223500. 
[48] P.M. Ferreira, A.E. Ruano, C. Fonseca, Evolutionary multi-objective design of radial basis function networks for greenhouse environmental control, in: The 16th IFAC World Congress on Automatic Control, vol. 16, Prague, Czech Republic, 2005, http://dx.doi.org/10.3182/20050703-6-CZ-1902.00871.

[49] P.M. Ferreira, A.E. Ruano, Application of computational intelligence methods to greenhouse environmental modelling, in: (WCCI 2008) IJCNN 2008 (IEEE World Congress on Computational Intelligence). 2008 IEEE International Joint Conference on Neural Networks, 2008, 2008, pp. 3582-3589, http://dx.doi.org/10.1109/IJCNN.2008.4634310.
[50] P.M. Ferreira, Application of computational intelligence methods to greenhouse environmental control, Ph.D. Thesis, Faculty of Sciences and Technology, University of Algarve, Faro, Portugal, 2008, January.

[51] J.M. Sousa, R. Babuška, H.B. Verbruggen, Fuzzy predictive control applied to an air-conditioning system, Control Engineering Practice 5 (10) (1997) 1395-1406.

[52] Mitsubishi Electric, How to Calculate the Electric Energy Consumption Ratio of City Multi by BMS via LMAP02, 2001. 